EXCITONIC EIGENSTATES OF

DISORDERED SEMICONDUCTOR QUANTUM WIRES:

ADAPTIVE WAVELET COMPUTATION OF EIGENVALUES FOR THE ELECTRON-HOLE SCHRÖDINGER EQUATION

By

Christian Mollet

Angela Kunoth

and

Torsten Meier

IMA Preprint Series \# 2381

( September 2011)

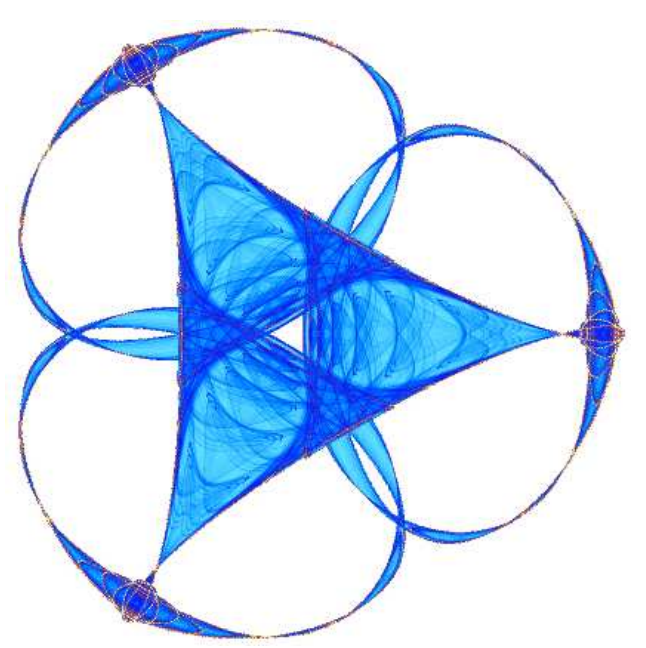

INSTITUTE FOR MATHEMATICS AND ITS APPLICATIONS

UNIVERSITY OF MINNESOTA

400 Lind Hall

207 Church Street S.E.

Minneapolis, Minnesota 55455-0436

Phone: 612/624-6066 Fax: 612/626-7370

URL: http://www.ima.umn.edu 


\title{
Excitonic Eigenstates of Disordered Semiconductor Quantum Wires: Adaptive Wavelet Computation of Eigenvalues for the Electron-Hole Schrödinger Equation
}

\author{
Christian Mollet ${ }^{1}$, Angela Kunoth ${ }^{1, *}$, and Torsten Meier ${ }^{2}$ \\ 1 Institut für Mathematik, Universität Paderborn, Warburger Str. 100, D-33098 \\ Paderborn, Germany. \\ ${ }^{2}$ Department Physik, Universität Paderborn, Warburger Str. 100, D-33098 Pader- \\ born, Germany.
}

\begin{abstract}
A novel adaptive approach to compute the eigenenergies and eigenfunctions of the two-particle (electron-hole) Schrödinger equation including Coulomb attraction is presented. As an example, we analyze the energetically lowest exciton state of a thin one-dimensional semiconductor quantum wire in the presence of disorder which arises from the non-smooth interface between the wire and surrounding material. The eigenvalues of the corresponding Schrödinger equation, i.e., the onedimensional exciton Wannier equation with disorder, correspond to the energies of excitons in the quantum wire. The wavefunctions, in turn, provide information on the optical properties of the wire.

We reformulate the problem of two interacting particles that both can move in one dimension as a stationary eigenvalue problem with two spacial dimensions in an appropriate weak form whose bilinear form is arranged to be symmetric, continuous, and coercive. The disorder of the wire is modelled by adding a potential in the Hamiltonian which is generated by normally distributed random numbers. The numerical solution of this problem is based on adaptive wavelets. Our scheme allows for a convergence proof of the resulting scheme together with complexity estimates. Numerical examples demonstrate the behavior of the smallest eigenvalue, the ground state energies of the exciton, together with the eigenstates depending on the strength and spatial correlation of disorder.
\end{abstract}

AMS subject classifications: 81Q05, 65N25, 65N50, 65Zxx.

Key words: Semiconductor quantum wire, disorder, Schrödinger equation, eigenvalue problem, weak formulation, bounded and coercive bilinear form, adaptive wavelet method, convergence, complexity, ground state energies, exciton. *Corresponding author. Email addresses: $\quad$ \{mollet,kunoth\}@math.uni-paderborn.de,
torsten.meier@uni-paderborn.de 


\section{Introduction}

Semiconductors and semiconductor nanostructures, e.g., wells, wires, and dots, play a prominent role in modern technology: computer chips are based on their electronic properties; the optical and optoelectronic properties of semiconductors are exploited in solar cells, light emitting diodes, and lasers, and, furthermore, future applications in the area of quantum communications and computing are expected to be within reach of current technology $[3,12,17,24]$. In this paper, we consider thin semiconductor quantum wires which are essentially one-dimensional systems, see Figure 1 . This approximation is appropriate if the diameter is much smaller than the intrinsic electronic length scale of the problem which is the exciton Bohr radius since we study the optical absorption close to the fundamental band gap. By optical excitation, one generates an electron-hole pair whose states are described by a two-particle time-dependent Schrödinger equation. Due to the unavoidable imperfections during the growth of such structures, the interface between the wire and the surrounding material cannot be considered to be perfectly smooth. We call this a disordered quantum wire and model it by an additional disorder potential in the Hamiltonian describing the spatial variation of the electron and hole energies. Thus, we analyze a model with diagonal disorder $[2,36]$, which has recently been used to study linear and nonlinear optical properties of semiconductor nanostructures together with a tight-binding model, see, e.g., [14,22,24,27]. From this, we formulate a stationary eigenvalue problem in two spatial dimensions describing the two interacting one-dimensional particles.

For the numerical solution, we seek for a highly efficient method, i.e., employing degrees of freedom for the computation and representation of eigenenergies and eigenstates only where actually needed. This paradigm has, from a numerical point of view, triggered much more substantial advancements in highly accurate simulations than increased computer power and larger storage systems. Thus, for us, it is indispensable to utilize a) an adaptive method which introduces during the computation and depending on the residuals of the operator equation and singularities of the problem additional degrees of freedom according to a user-specified accuracy. In view of extensions to quantum films and a resulting partial differential operator in four space dimensions to be considered at a later stage, we want to ensure that our method could b) systematically be adapted to higher spatial dimensions. In addition, it is important to us to c) be able to prove convergence of the numerical method. This means that an addition of degrees of freedom provably reduces the numerical error. Lastly, we want to assure that d) our method provides optimal computational complexity. This means that the algorithm has an optimal work/accuracy balance, in a sense specified below.

In view of all of these requests, for the numerical solution of the eigenvalue problem, adaptive methods based on finite elements like in [23], finite differences or spectral methods are excluded. Our method of choice are adaptive wavelets for which convergence and complexity results can be expected, in view of the paradigm of an adaptive wavelet method for stationary linear variational partial differential equations. These re- 
sults were developed firstly in [5]. Recall that wavelets provide a Riesz basis for a whole range of Sobolev spaces, allowing us to represent functions belonging to these spaces as infinite vectors, i.e., their coefficients in a wavelet expansion. Similarly, operator equations defined on Hilbert spaces can be represented in terms of infinite vectors. Thus, initially, one can stay as close as possible to the functional analytic formulation of the problem. Moreover, an appropriate scaling of wavelet coefficient vectors ensures wellconditioned systems in the $\ell_{2}$ topology, thereby ensuring fast convergence of iterative methods. Now computational routines can be developed which mimic the application of biinfinite linear operators. It is characteristic for this approach that the adaptive scheme is solely governed by the desired final accuracy; any a-priori user-selected finest level of resolution is not required. This strategy not only ensures convergence of the adaptive scheme: one is able to prove optimal complexity of the solution when compared to its wavelet-best $N$-term approximation. This means the following: if the exact solution was known, one could ask for the best possible approximation with a prescribed number of degrees of freedom $N$. Given the regularity of the solution in certain Besov spaces which are much larger smoothness classes than Sobolev spaces and which allow, e.g., for isolated singularities, one can prove that the wavelet-best $N$-approximation can be achieved with a rate depending on this regularity. Moreover, the method exhibits an optimal work/accuracy balance meaning that the amount of arithmetic operations to compute the solution is proportional to $N$. For general surveys on the application of wavelets to operator equations, we refer to [4,8], and to [11] for the newest state of research on this topic. For the time-dependent Schrödinger equation, a collocation method on uniform grids employing Daubechies' orthogonal scaling functions has been proposed recently, see [35] and the original article it refers to.

The idea of solving eigenvalue problems with a linear elliptic operator employing adaptive wavelets has been proposed in [9] together with a convergence proof and complexity estimates. Here we follow to some extent an improved version of the algorithm from [31]. For many details concerning the material in this paper, we refer to [26], see also [21].

The remainder of this paper is organized as follows. In the next section, we describe the physical model. We derive the resulting eigenvalue problem in a weak form in Section 3 and show boundedness and coercivity of an appropriately shifted bilinear form. Moreover, we prove a certain decay property of the involved operators which later allow the approximate application of the operators in wavelet coordinates. The necessary ingredients of wavelets are collected in Section 4 which are then applied to the eigenvalue problem in Section 5. Section 6 contains various numerical results. We conclude in Section 7 with a short summary and some outlook. In the future, we plan to extend our adaptive approach to be applicable to multi-scale time-dependent problems. 


\section{The Physical Model}

The descriptions in this section concerning semiconductor nanostructures and their properties are only very rough since we wish to focus on the numerical solution scheme. Thus, the model introduced here shall only serve as a model problem. For extensive and detailed information and further problem formulations concerning optical and electrical properties of semiconductors, we refer to [17,24,27].

We consider a disordered semiconductor quantum wire, see Figure 1. This is essentially a one-dimensional semiconductor nanostructure which is made of two semiconductor materials with different band gaps such that the free electron motion is confined to one space dimensions. The wire consists of the semiconductor material SCM1 and the direct neighborhood of the other semiconductor material SCM2. In this neighborhood, there is a higher potential implying that the electrons can only move in the one-dimensional horizontal direction since they cannot leave the semiconductor material SCM1. Because of production restrictions, one cannot assume that the interface between the two semiconductors is perfectly plain or smooth. These fluctuations of the interface are called disorder and lead to a disorder potential on the interface. The physical effect of optical absorption

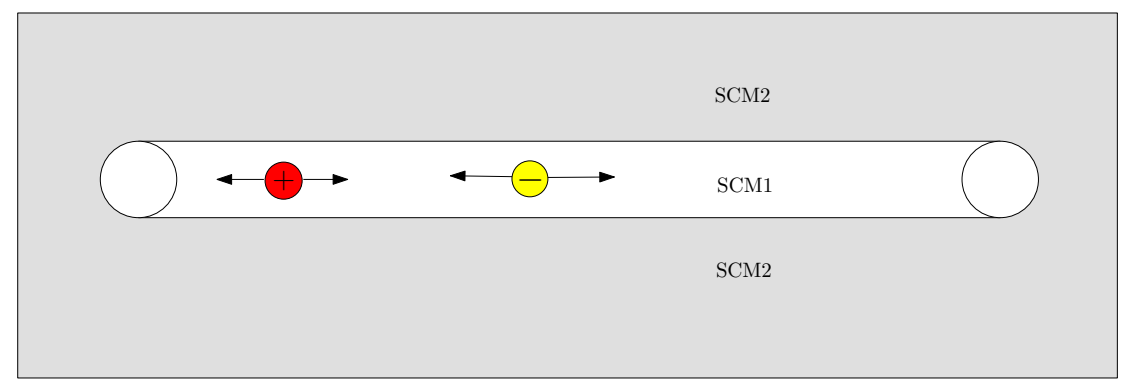

Figure 1: Schematical drawing of a semiconductor quantum wire. The wire itself consists of a semiconductor material SCM1 which is surrounded by another semiconductor material SCM2 which has a higher band gap than SCM1. As a result, the electron (yellow) and the hole (red) can move in one dimension only.

in semiconductors can be explained with the aid of a so-called energy band model. Here, we restrict our analysis to a two band model and include one valence band and one conduction band in our analysis. Between these two bands there is a valence-conduction band gap of width $E_{g}=E_{c}-E_{v}$ where $E_{v}$ is the highest possible energy in the valence band and $E_{c}$ the lowest energy in the conduction band. If the valence band is completely filled and the conduction band is empty, no current is flowing if a weak static electric field is applied. This state is the ground state, i.e., the state with the smallest energy of the system, of the intrinsic (undoped) semiconductor.

The effect described next is utilized for power generation with the aid of, e.g., solar cells. An optical excitation by light of a frequency $\omega$ with $\hbar \omega>E_{g}$ causes an increase 
of energy of an electron in the valence band and lifts it over the energy gap into the conduction band, thereby causing an interband transition. Here $\hbar=1,054 \times 10^{-34} \mathrm{Js}=$ $6,582 \times 10^{-16} \mathrm{eVs}$ is Planck's constant. Consequently, the conduction band is no longer empty: there is a movable electron inside. Because the electron is no longer located in the valence band, a so-called hole has been created at this position. This hole may be viewed as an imaginary positively charged particle which explains the attraction between the electron and the hole. This process is called electron-hole pair generation. A bound electronhole pair is also called exciton.

The main difference between a semiconductor, an insulator and a metal is the value of the factor $E_{g}$. In an insulator, this energy gap is very high such that, in principle, an electron cannot jump over this gap. In a metal, this gap does not exist or is close to zero. Since there is no explicit value of the energy gap $E_{g}$ which clearly classifies materials, one usually does not distinguish between semiconductors and insulators.

The model yields a time-dependent two-particle Schrödinger equation with an optical excitation, i.e.,

$$
\begin{array}{r}
i \hbar \frac{\partial}{\partial t} p\left(x_{e}, x_{h}, t\right)=\left(E_{g}+\hat{H}_{k i n}+\hat{H}_{a t t r}+\hat{H}_{d i s}\right) p\left(x_{e}, x_{h}, t\right) \\
-\hat{\mu} E\left(x_{h}, t\right) \delta\left(x_{e}-x_{h}\right),
\end{array}
$$

which describes the (complex-valued) state function $p\left(x_{e}, x_{h}, t\right)$ of the electron-hole pair. Here

$$
\hat{H}_{k i n}:=-\frac{\hbar^{2}}{2 m_{e}^{*}} \frac{\partial^{2}}{\partial x_{e}^{2}}-\frac{\hbar^{2}}{2 m_{h}^{*}} \frac{\partial^{2}}{\partial x_{h}^{2}}
$$

denotes the Hamiltonian operator of two free particles,

$$
\hat{H}_{a t t r}:=\frac{-e^{2}}{4 \pi \hat{\varepsilon}_{0} \hat{\varepsilon}_{r}\left(\min \left\{\left|x_{e}-x_{h}\right|,\left|x_{e}-x_{h} \pm L\right|\right\}+\hat{\gamma} \hat{R}\right)}
$$

describes the electron-hole attraction and

$$
\hat{H}_{d i s}=V_{d i s, e}\left(x_{e}\right)+V_{d i s, h}\left(x_{h}\right)
$$

models the disorder of the interface of the wire which will be specified below in (2.8). The term

$$
-\mu E\left(x_{h}, t\right) \delta\left(x_{e}-x_{h}\right)
$$

describes the optical excitation where the Dirac delta $\delta(x)$ models the excitation and $E\left(x_{h}, t\right)$ denotes the function of the electric field of the optical excitation, i.e., the electric field of the incident light. In (2.2), $m_{e}^{*}$ is the effective mass of an electron and $m_{h}^{*}$ the effective mass of a hole. Moreoever, in (2.3), $e=1 e=1,602 \times 10^{-19} \mathrm{C}$ is the elementary charge, $\hat{\varepsilon}_{0}=8,854 \times 10^{-12} \frac{\mathrm{C}}{V m}$ is the electric constant or vacuum permittivity, $\hat{\varepsilon}_{r}$ the relative permittivity and $\hat{\gamma} \hat{R}$ a regularization parameter. In (2.5), $\hat{\mu}$ denotes the optical dipole-matrix-element. The length $L>0$ of the quantum wire appearing in (2.3) and also 
its diameter which in turn defines the regularization parameter $\hat{\gamma} \hat{R}$ is determined by the type of wire.

The electronic Schrödinger equation (2.1) is a (complex-valued) partial differential equation (PDE) formulated over a domain $(0, L)^{2} \times I$ where $I:=\left[t_{0}, \hat{T}\right] \subseteq \mathbb{R}$ denotes the time interval with fixed initial time $t_{0}$ and (flexible) end time $\hat{T}$. We equip the PDE (2.1) with periodic boundary conditions

$$
\begin{aligned}
& p\left(0, x_{h}, t\right)=p\left(L, x_{h}, t\right), \quad \text { for all } x_{h} \in[0, L], t \in I, \\
& p\left(x_{e}, 0, t\right)=p\left(x_{e}, L, t\right), \quad \text { for all } x_{e} \in[0, L], t \in I \text {, }
\end{aligned}
$$

and the same for the first derivatives of $p$, and zero initial conditions

$$
p\left(x_{e}, x_{h}, t_{0}\right)=0 \quad \text { for all }\left(x_{e}, x_{h}\right) \in(0, L)^{2} .
$$

The minimum in $\hat{H}_{\text {attr }}$ reflects the periodic model for the Schrödinger equation since the Coulomb interaction is determined by the smallest distance $\left|x_{e}-x_{h}\right|$ or $\left|x_{e}-x_{h} \pm L\right|$. In many cases, one has a homogeneous optical excitation such that the electric field only depends on time, i.e., $E\left(x_{h}, t\right)$ simplifies to $E(t)$. This is the case, for example, when $E(t) \sim \exp \left(-(t / \hat{\tau})^{2}\right)$ with a constant parameter $\hat{\tau} \in \mathbb{R}$.

In the case of an ideal wire, i.e., a quantum wire with a completely straight interface, the expression $\hat{H}_{d i s}$ vanishes. However, due to production processes involving a random disorder of the interface with the surrounding material, we have to deal with a non-ideal wire. The potential functions $V_{d i s, h}$ and $V_{d i s, e}$ appearing in (2.4) may therefore be assumed to be a periodic potential function on $(0, L)$ or, more realistically, may be modelled as a stochastic perturbation on $(0, L)$. This we describe using a piecewise constant function with randomly chosen step heights,

$$
V_{d i s, e}\left(x_{e}\right):=\sum_{i=1}^{M} \operatorname{Ran}_{d i s, e}(i) \operatorname{Char}_{\left[(i-1) \frac{L}{M}, i \frac{L}{M}\right)}\left(x_{e}\right),
$$

where $\operatorname{Char}_{\hat{I}}\left(x_{e}\right):=1$ for $x_{e} \in \hat{I}$ and zero otherwise denotes the characteristic function on an interval $\hat{I}$ and $M$ is the number of steps. Furthermore, $\operatorname{Ran}_{d i s, e}(i) \sim \mathcal{N}\left(0, \sigma^{2}\right)$ for all $i \in \mathbb{N}$ are the corresponding randomly chosen step heights, that is, $\operatorname{Ran}_{d i s, e}(i)$ is for each $i \in \mathbb{N}$ a normally distributed random number with expectation zero and variance $\sigma^{2}$. Expectation zero states that the fluctuations of the interface are uniform in the sense that the average deviation of the thickness with respect to the ideal wire with the corresponding energy gap $E_{g}$ is zero. Moreover, the size of the variance describes the amount of the probability for large deviations, meaning that the variance is a quantity for the size of the stochastic perturbation. One can also use a moving or sliding average of the data set.

The explicit realization of the disorder potentials, that is, of the random numbers, is performed for our numerical experiments following the recipes provided in [30], see [26] for details: first we generate uniformly distributed random numbers on the interval $(0,1)$ using Marsaglia's 64-bit Xorshift random number generator in combination with 
a multiply-with-carry and a linear congruential random number generator. The authors of [30] call this combination "our suspender-and-belt, full-body-armor, never-any-doubt generator". We have employed this up-to-date perhaps most sophisticated choice since we wanted to assure that the randomness of the wire interfaces resembles to a high amount the actual production process. Afterwards, we employ the Box-Muller method to derive standard normally distributed random numbers with expectation zero and variance one. Finally, multiplying these numbers by $\sigma$ yields normally distributed random numbers $\sim \mathcal{N}\left(0, \sigma^{2}\right)$.

The disorder potential for the hole $V_{d i s, h}\left(x_{h}\right)$ can be chosen analogously, or it can be defined as $V_{d i s, h}\left(x_{h}\right):=\frac{m_{e}^{*}}{m_{h}^{*}} V_{d i s, e}\left(x_{e}\right)$ which means that one only has to generate one of the disorder potentials. This so-called correlated disorder will be employed in the sequel.

\section{The Eigenvalue Problem}

\subsection{Classical Formulation}

Next we formulate the PDE (2.1) as an eigenvalue problem using a standard separation of variables approach; its detailed derivation can be found in [26].

Proposition 3.1. Consider the time independent part of the Hamiltonian and assume that the state function $p\left(x_{e}, x_{h}, t\right)$ can be expressed as a product

$$
p\left(x_{e}, x_{h}, t\right)=X(x) T(t), \quad x:=\left(x_{e}, x_{h}\right)^{T},
$$

for all $(x, t) \in(0, L)^{2} \times I$. Then the instationary Schrödinger equation (2.1) implies the stationary eigenvalue equation

$$
\hat{E} X(x)=\hat{H} X(x), \quad x \in(0, L)^{2},
$$

where $\hat{E} \in \mathbb{C}$ is constant and the Hamilton operator

$$
\hat{H}:=E_{g}+\hat{H}_{k i n}+\hat{H}_{a t t r}+\hat{H}_{d i s}
$$

is the time independent part of the Schrödinger equation (2.1).

Note that we can write $\hat{H}(X(x))=\hat{H} X(x)$ since $\hat{H}$ is a linear operator. The eigenvalues of (3.2) can be interpreted as the energy of the system in the state described by the state function, i.e., the energy of the electron-hole pair. Physically relevant are the lowest energies, that means, the smallest eigenvalues. Moreover, the probability density is given by $|X(x)|^{2}$. These quantities are interesting from a quantum-physical point of view: with their aid, one can determine the probability to find an exciton with a certain energy given by the eigenvalues of (3.2) in a certain area of the quantum wire.

At this point, we would like to mention that it is possible to express the timedependent solution $p(x, t)$ in terms of a sum over the the eigenfunctions $X(x)$ multiplied with coefficients and the corresponding time-dependent oscillatory exponential, 
see [17]. In the following, we will restrict ourselves to compute the smallest eigenvalues and the corresponding eigenfunctions of the eigenvalue problem with periodic boundary conditions(3.2). Extensions, i.e., the computatation of energetically higher eigenfunctions are possible if one projects out the space spanned by the energetically lower eigenfunctions.

In [26], the stationary eigenvalue problem (3.2) was rewritten into (Hartree) atomic units. In these units, the length is expressed in terms of the Bohr radius with respect to a hydrogen atom $a_{0}=5,292 \times 10^{-11} \mathrm{~m}$ and the energy in terms of the corresponding Hartree Energy $E_{h}=4,360 \times 10^{-18} \mathrm{~J}$.

\subsection{Weak Formulation}

Our numerical solution will be based on an appropriate weak form of (3.2). Recall from, e.g., [10] that weak or variational forms involving elliptic PDE operators typically require less smoothness of the solution than the classical strong form (3.2) in order to establish convergence results of discretizations. Following [34], we can, in particular, for the weak form derive properties on the spectrum of operators defined via the bilinear form (3.7) below which will be essential for the solution scheme employed later.

In view of the periodic boundary conditions (2.6) of the problem (3.2), we will employ as test and trial spaces a periodic Sobolev space using the space $\mathcal{C}_{\text {per }}^{\infty}\left([0, L]^{2}\right)$ of infinitely many times differentiable functions which are periodic on $[0, L]^{2}$, that is, all their derivatives at 0 coincide with the derivatives at $L$ in each variable. We then define the periodic Sobolev space of order one as the closure of this space with respect to the norm of the classical Sobolev space $H^{1}(\Omega)$ on the domain $\Omega:=(0, L)^{2}$, i.e., $H_{p e r}^{1}(\Omega):=$ $\operatorname{clos}_{\|\cdot\|_{H^{1}(\Omega)}}\left(\mathcal{C}_{\text {per }}^{\infty}(\bar{\Omega})\right)$. Recall from, e.g., [1] on detailed definitions and information on Sobolev spaces that this is equivalent to defining $H_{\text {per }}^{1}(\Omega):=\left\{v \in H^{1}(\Omega): v\left(0, x_{h}\right)=\right.$ $v\left(L, x_{h}\right)$ for all $x_{h} \in[0, L]$ and $v\left(x_{e}, 0\right)=v\left(x_{e}, L\right)$ for all $\left.x_{e} \in[0, L]\right\}$ (considering equalities in the sense of traces). Using these spaces, problem (3.2) subject to (2.6) can be expressed in the following weak form: find $u \in H_{\text {per }}^{1}(\Omega)$ and $\hat{E} \in \mathbb{C}$ such that

$$
\hat{a}(u, v)=\hat{E}(u, v)_{L_{2}(\Omega)} \quad \text { for all } v \in H_{\text {per }}^{1}(\Omega),
$$

where $(u, v)_{L_{2}(\Omega)}:=\int_{\Omega} u(x) v(x) d x$ is the standard inner product for the Lebesgue space $L_{2}(\Omega)$ with Lebesgue measure $d x$ and inducing the $L_{2}(\Omega)$-norm $\|v\|_{L_{2}(\Omega)}^{2}:=$ $\int_{\Omega} v(x) v(x) d x$. Moreover, $\hat{a}: H_{\text {per }}^{1}(\Omega) \times H_{\text {per }}^{1}(\Omega) \rightarrow \mathbb{R}$ is the bilinear form defined by

$$
\begin{aligned}
\hat{a}(u, v):= & E_{g}(u, v)_{L_{2}(\Omega)} \\
& +\frac{\hbar^{2}}{2 m_{e}^{*}}\left(\frac{\partial}{\partial x_{e}} u, \frac{\partial}{\partial x_{e}} v\right)_{L_{2}(\Omega)}+\frac{\hbar^{2}}{2 m_{h}^{*}}\left(\frac{\partial}{\partial x_{h}} u, \frac{\partial}{\partial x_{h}} v\right)_{L_{2}(\Omega)} \\
& -\int_{\Omega} \frac{e^{2}}{4 \pi \hat{\varepsilon}_{0} \hat{\varepsilon}_{r}\left(\min \left\{\left|x_{e}-x_{h}\right|,\left|x_{e}-x_{h} \pm L\right|\right\}+\hat{\gamma} \hat{R}\right)} u(x) v(x) d x
\end{aligned}
$$




$$
\begin{aligned}
& +\sum_{i=1}^{M} \operatorname{Ran}_{d i s, e}(i) \int_{0}^{L} \int_{(i-1) \frac{L}{M}}^{i \frac{L}{M}} u(x) v(x) d x_{e} d x_{h} \\
& +\sum_{i=1}^{M} \operatorname{Ran}_{d i s, h}(i) \int_{(i-1) \frac{L}{M}}^{i \frac{L}{M}} \int_{0}^{L} u(x) v(x) d x_{e} d x_{h} .
\end{aligned}
$$

In order to derive that the spectrum of the operator in (3.4) is strictly positive which is a comfortable property for solving (3.4), one condition requires that the bilinear form $\hat{a}(\cdot, \cdot)$ is coercive on $H_{\text {per }}^{1}(\Omega)$ which it is not in this form. However, we can achieve this by shifting both sides of (3.4) as follows: problem (3.4) is equivalent to the problem to find $u \in H_{\text {per }}^{1}(\Omega)$ and $\hat{E} \in \mathbb{C}$ such that

$$
a(u, v)=\left(\hat{E}+E_{s}\right)(u, v)_{L_{2}(\Omega)} \quad \text { for all } v \in H_{p e r}^{1}(\Omega),
$$

with

$$
a(u, v):=\hat{a}(u, v)+E_{S}(u, v)_{L_{2}(\Omega)}
$$

with a constant shift parameter $E_{s} \in \mathbb{R}$ chosen such that

$$
E_{S}>-\min \left\{E_{g},-\frac{e^{2}}{4 \pi \hat{\varepsilon}_{0} \hat{\varepsilon}_{r} \hat{\gamma} \hat{R}^{\prime}}{ }_{i=1, \ldots, M} \min _{\text {is }}\left\{\operatorname{Ran}_{d i s, e}(i), \operatorname{Ran}_{d i s, h}(i)\right\}\right\}>0 .
$$

Then we can establish the following theoretical result whose proof also reveals the choice of the shift (3.8).

Theorem 3.2. For a fixed given realization of the random terms $\operatorname{Ran}_{d i s, e}$ and $\operatorname{Ran}_{d i s, h}$, the bilinear form $a(\cdot, \cdot)$ defined in (3.7) is symmetric, bounded and coercive on $H_{\text {per }}^{1}(\Omega)$ : there exist some constants $0<c_{A} \leq C_{A}<\infty$ such that

$$
\begin{array}{lrl}
a(u, v) & \leq C_{A}\|u\|_{H_{p e r}^{1}(\Omega)}\|v\|_{H_{p e r}^{1}(\Omega),} & u, v \in H_{p e r}^{1}(\Omega), \\
a(u, u) \geq c_{A}\|u\|_{H_{p e r}^{1}(\Omega)}^{2}, & u \in H_{p e r}^{1}(\Omega),
\end{array}
$$

employing $\|v\|_{H_{p e r}^{1}(\Omega)}^{2}:=(v, v)_{L_{2}(\Omega)}+(\nabla v, \nabla v)_{L_{2}(\Omega)}$.

Proof. For a fixed given realization of the random terms $\operatorname{Ran}_{d i s, e}$ and $\operatorname{Ran}_{d i s, h}$, we immediately see by inspection that $a(u, v)=a(v, u)$ for all $u, v \in H_{\text {per }}^{1}(\Omega)$.

The boundedness of $a(\cdot, \cdot)$ can be shown by estimating each term in a straightforward fashion. First observe that

$$
\begin{aligned}
& -\int_{\Omega} \frac{e^{2} u\left(x_{e}, x_{h}\right) v\left(x_{e}, x_{h}\right)}{4 \pi \hat{\varepsilon}_{0} \hat{\varepsilon}_{r}\left(\min \left\{\left|x_{e}-x_{h}\right|,\left|x_{e}-x_{h} \pm L\right|\right\}+\hat{\gamma} \hat{R}\right)} d x \\
& \quad \leq-\frac{e^{2}}{4 \pi \hat{\varepsilon}_{0} \hat{\varepsilon}_{r}(L / 2+\hat{\gamma} \hat{R})} \int_{\Omega} u\left(x_{e}, x_{h}\right) v\left(x_{e}, x_{h}\right) d x \\
& \quad \leq \int_{\Omega} u\left(x_{e}, x_{h}\right) v\left(x_{e}, x_{h}\right) d x
\end{aligned}
$$


since $\min _{x_{e}, x_{h} \in(0, L)}\left\{\left|x_{e}-x_{h}\right|,\left|x_{e}-x_{h} \pm L\right|\right\} \leq L / 2$ and $\hat{\varepsilon}, \hat{\varepsilon}_{0}, L, \hat{\gamma} \hat{R}>0$. Next, for a fixed realization of $\operatorname{Ran}_{d i s, e}$ and $\operatorname{Ran}_{d i s, h}$, we have

$$
\begin{gathered}
\int_{\Omega} \sum_{i=1}^{M} \operatorname{Ran}_{d i s, e}(i) \operatorname{Char}_{\left[(i-1) \frac{L}{M}, i \frac{L}{M}\right)}\left(x_{e}\right) u\left(x_{e}, x_{h}\right) v\left(x_{e}, x_{h}\right) d x \\
\quad \leq \max _{i=1, \ldots, M}\left\{\operatorname{Ran}_{d i s, e}(i)\right\} \int_{\Omega} u\left(x_{e}, x_{h}\right) v\left(x_{e}, x_{h}\right) d x .
\end{gathered}
$$

In view of the definition (3.7) and putting (3.11), (3.12) together, we arrive at

$$
\begin{aligned}
a(u, v)= & \hat{a}(u, v)+E_{s}(u, v)_{L_{2}(\Omega)} \\
\leq & \max \left\{E_{g,}, \frac{\hbar^{2}}{2 m_{e}^{*}}, \frac{\hbar^{2}}{2 m_{e}^{*}}, \max _{i=1, \ldots, M}\left\{\left|\operatorname{Ran}_{d i s, e}(i)\right|,\left|\operatorname{Ran}_{d i s, h}(i)\right|\right\}, 1\right\} \\
& \times \int_{\Omega}\left(4 u v+\left(\frac{\partial}{\partial x_{e}} u\right)\left(\frac{\partial}{\partial x_{e}} v\right)+\left(\frac{\partial}{\partial x_{h}} u\right)\left(\frac{\partial}{\partial x_{h}} v\right)\right) d x+E_{s}(u, v)_{L_{2}(\Omega)} \\
\lesssim & \int_{\Omega}(u v+\nabla u \cdot \nabla v) d x=(u, v)_{H_{p e r}^{1}(\Omega)} \leq\|u\|_{H_{p e r}^{1}(\Omega)}\|v\|_{H_{p e r}^{1}(\Omega)}
\end{aligned}
$$

i.e., the bilinear form is bounded (3.9) for any $u, v \in H_{\text {per }}^{1}(\Omega)$. Here we have omitted for better readability the dependencies in $u=u\left(x_{e}, x_{h}\right), v=v\left(x_{e}, x_{h}\right)$, and the abbreviation $b \lesssim c$ means that $b$ can be bounded from above by a constant multiple of $c$.

As for the coercivity, we first estimate the unshifted bilinear form $\hat{a}(\cdot, \cdot)$ defined in (3.5) from below. To this end, observe that

$$
\begin{gathered}
-\int_{\Omega} \frac{e^{2} u\left(x_{e}, x_{h}\right)^{2}}{4 \pi \hat{\varepsilon}_{0} \hat{\varepsilon}_{r}\left(\min \left\{\left|x_{e}-x_{h}\right|,\left|x_{e}-x_{h} \pm L\right|\right\}+\hat{\gamma} \hat{R}\right)} d x \\
\geq-\frac{e^{2}}{4 \pi \hat{\varepsilon}_{0} \hat{\varepsilon}_{r} \hat{\gamma} \hat{R}} \int_{\Omega} u\left(x_{e}, x_{h}\right)^{2} d x
\end{gathered}
$$

since $\min \left\{\left|x_{e}-x_{h}\right|,\left|x_{e}-x_{h} \pm L\right|\right\}+\hat{\gamma} \hat{R} \geq 0$. Thus, we obtain

$$
\begin{aligned}
\hat{a}(u, u) \geq \min & \left\{E_{g},-\frac{e^{2}}{4 \pi \hat{\varepsilon}_{0} \hat{\varepsilon}_{r} \hat{\gamma} \hat{R}^{\prime}} \min _{i=1, \ldots, M}\left\{\operatorname{Ran}_{\text {dis,e }}(i), \operatorname{Ran}_{d i s, h}(i)\right\}\right\} \\
\times & \int_{\Omega} 4 u^{2} d x+\min \left\{\frac{\hbar^{2}}{2 m_{e}^{*},}, \frac{\hbar^{2}}{2 m_{e}^{*}}\right\} \int_{\Omega}\left(\left(\frac{\partial}{\partial x_{e}} u\right)^{2}+\left(\frac{\partial}{\partial x_{h}} u\right)^{2}\right) d x \\
\geq \min & \left\{E_{g},-\frac{e^{2}}{4 \pi \hat{\varepsilon}_{0} \hat{\varepsilon}_{r}+\hat{\gamma}^{2}}, \frac{\hbar^{2}}{2 m_{e}^{*}}, \frac{\hbar^{2}}{2 m_{h}^{*}}, \min _{i=1, \ldots, M}\left\{\operatorname{Ran}_{d i s, e}(i), \operatorname{Ran}_{d i s, h}(i)\right\}\right\} \\
& \times \int_{\Omega}\left(4 u^{2}+\left(\frac{\partial}{\partial x_{e}} u\right)^{2}+\left(\frac{\partial}{\partial x_{h}} u\right)^{2}\right) d x .
\end{aligned}
$$

We see here that the constant bounding $\hat{a}(\cdot, \cdot)$ from below is negative so that the bilinear form $\hat{a}(\cdot, \cdot)$ is not coercive. Such kind of estimates with a possibly negative constant with respect to the $L_{2}(\Omega)$-norm are also considered in a slightly different context of PDEs in 
connection with the Gårding inequality, see e.g., [15] from which we borrow the following idea. We reformulate the corresponding eigenvalue problem by shifting the left and right hand side by a suitable constant times $(u, v)_{L_{2}(\Omega)}$. To this end, note that the eigenvalue problem (3.4) is equivalent to the eigenvalue problem (3.6) with a constant shift parameter $E_{s} \in \mathbb{R}$. Therefore, shifting $\hat{a}(\cdot, \cdot)$ with a fixed constant $E_{s}$ such that $E_{s}$ satisfies (3.8) yields

$$
\begin{aligned}
a(u, u)= & \hat{a}(u, u)+E_{s}(u, u)_{L_{2}(\Omega)} \\
\geq & \left(\min \left\{E_{g},-\frac{e^{2}}{4 \pi \hat{\varepsilon}_{0} \hat{r}_{r} \hat{\gamma} \hat{R}^{\prime}} \min _{i=1, \ldots, M}\left\{\operatorname{Ran}_{\text {dis,e }}(i), \operatorname{Ran}_{d i s, h}(i)\right\}\right\}+E_{s}\right) \\
& \quad \times \int_{\Omega} 4 u^{2} d x+\min \left\{\frac{\hbar^{2}}{2 m_{e}^{*},}, \frac{\hbar^{2}}{2 m_{h}^{*}}\right\} \int_{\Omega}\left(\left(\frac{\partial}{\partial x_{e}} u\right)^{2}+\left(\frac{\partial}{\partial x_{h}} u\right)^{2}\right) d x \\
\geq & C \int_{\Omega}\left(4 u^{2}+\left(\frac{\partial}{\partial x_{h}} u\right)^{2}\right) d x \\
\geq & \int_{\Omega}\left(u^{2}+(\nabla u)^{2}\right) d x=\|u\|_{H^{1}(\Omega)^{\prime}}^{2}
\end{aligned}
$$

where the involved constant $C>0$ is defined as

$$
C:=\min \left\{c_{1}, c_{2}\right\}>0
$$

with

$$
c_{1}:=\min \left\{E_{g},-\frac{e^{2}}{4 \pi \hat{\varepsilon}_{0} \hat{\varepsilon}_{r} \hat{\gamma} \hat{R}^{\prime}}{ }_{i=1, \cdots, M}\left\{\operatorname{man}_{d i s, e}(i), \operatorname{Ran}_{d i s, h}(i)\right\}\right\}+E_{s}
$$

and

$$
c_{2}:=\min \left\{\frac{\hbar^{2}}{2 m_{e}^{*}}, \frac{\hbar^{2}}{2 m_{h}^{*}}\right\} .
$$

Thus, the bilinear form $a(\cdot, \cdot)$ with respect to the reformulated eigenvalue problem (3.6) is now coercive on $H_{\text {per }}^{1}(\Omega)$.

The appearing function spaces are chosen such that we have the Gelfand triple

$$
H_{\text {per }}^{1}(\Omega) \hookrightarrow L_{2}(\Omega) \cong\left(L_{2}(\Omega)\right)^{\prime} \hookrightarrow H_{\text {per }}^{-1}(\Omega),
$$

where $\hookrightarrow$ means continuous embedding, i.e., there exists a constant $c_{e}>0$ such that

$$
\|v\|_{L_{2}(\Omega)} \leq c_{e}\|v\|_{H^{1}(\Omega)} \quad \text { for any } v \in H^{1}(\Omega),
$$

and the pivot space $L_{2}(\Omega)$ is identified with its dual $\left(L_{2}(\Omega)\right)^{\prime}$. Even stronger, the embedding $H_{\text {per }}^{1}(\Omega) \hookrightarrow L_{2}(\Omega)$ can be shown to be compact [1]. Together with the fact that, according to Theorem 3.2, the bilinear form $a(\cdot, \cdot)$ defined in (3.7) is symmetric, bounded and coercive on $H^{1}(\Omega)$, we can conclude the following result [34].

Corollary 3.3. All eigenvalues of $a(\cdot, \cdot)$ are real, strictly positive and bounded from below by $c_{a} / c_{e}^{2}>0$. Moreover, the lower part of the spectrum is discrete, meaning that it is a set of isolated eigenvalues of finite multiplicity, and all other eigenvalues accumulate at infinity. 
The following result is an immediate consequence of Riesz' representation theorem together with the definition of operator norms, see, e.g., [15].

Corollary 3.4. The bilinear form $a(\cdot, \cdot)$ uniquely defines a linear operator $A: H_{\text {per }}^{1}(\Omega) \rightarrow$ $\left(H_{\text {per }}^{1}(\Omega)\right)^{\prime}$ by $\langle A u, v\rangle:=a(u, v)$ for $u, v \in H_{\text {per }}^{1}(\Omega)$ where $\langle\cdot, \cdot\rangle$ denotes the dual pairing between the topological dual $H_{\text {per }}^{-1}(\Omega)$ of $H_{\text {per }}^{1}(\Omega)$ and $H_{\text {per }}^{1}(\Omega)$. Moreover, the operator $A$ is self-adjoint, bounded and positive on $H_{\text {per }}^{1}(\Omega)$ with the same constants $c_{A}$ and $C_{A}$ from (3.9), (3.10) so that $A$ satisfies the mapping property

$$
c_{A}\|u\|_{H_{p e r}^{1}(\Omega)} \leq\|A u\|_{H_{p e r}^{-1}(\Omega)} \leq C_{A}\|u\|_{H_{p e r}^{1}(\Omega)} \quad \text { for any } u \in H_{\text {per }}^{1}(\Omega),
$$

i.e., $A$ is boundedly invertible, written shortly as

$$
\|A u\|_{H_{p e r}^{-1}(\Omega)} \sim\|u\|_{H_{p e r}^{1}(\Omega)} \quad \text { for any } u \in H_{p e r}^{1}(\Omega) .
$$

Next we briefly derive a suitable operator form of (3.6). By Riesz' representation theorem, the inner product $(\cdot, \cdot)_{L_{2}(\Omega)}$ also uniquely defines a linear operator $R: L_{2}(\Omega) \rightarrow$ $\left(L_{2}(\Omega)\right)^{\prime}$ by $\langle R u, v\rangle:=(u, v)_{L_{2}(\Omega)}$ for $u, v \in L_{2}(\Omega)$ (with the corresponding dual form) called a Riesz operator. For an appropriate operator form of (3.6), we need the restriction $\left.R\right|_{H_{p e r}^{1}(\Omega)}: H_{\text {per }}^{1}(\Omega) \rightarrow H_{\text {per }}^{-1}(\Omega)$. Due to the continuous embedding (3.16), $\left.R\right|_{H_{\text {per }}^{1}(\Omega)}$ is bounded as an operator defined on $\left.R\right|_{H_{p e r}^{1}(\Omega)}$ with values in $H_{\text {per }}^{-1}(\Omega)$ as well. For simplicity, we denote the restriction $\left.R\right|_{H_{p e r}^{1}(\Omega)}$ again by $R$.

In the following, we will use capital letters to denote operators like $A, R$, and we exchange the letter denoting the eigenvalue $\hat{E}$ in (3.2) by $\mu$. We can formulate the (weak) eigenvalue problem (3.6) in terms of operators as follows: find the smallest eigenvalue $\mu \in \mathbb{C}$ and corresponding eigenfunction $u \in H_{\text {per }}^{1}(\Omega) \backslash\{0\}$ such that

$$
A u=\mu R u .
$$

The eigenvalue solution scheme in [31] is formulated for this operator equation on infinite Hilbert spaces. Our explicit realization of this method will be expressed below in terms of wavelets in Section 4.2 .

\section{Wavelets}

\subsection{Basic Properties}

We shall assume that for the underlying Hilbert space $\mathcal{H}:=H_{\text {per }}^{1}(\Omega)$ we have at our disposal a collection of functions, a wavelet basis,

$$
\Psi=\left\{\psi_{\lambda}: \lambda \in \mathbb{I}\right\} \subset \mathcal{H}
$$

with the following properties. II is an infinite index set whose elements $\lambda$ represent indices $(j, \mathbf{k}, \mathbf{e})$ where $j:=|\lambda|$ stands for a scale of resolution or refinement level, $k$ denotes 
the spatial location and e classifies the type of (tensor-product) basic wavelet. Instead of giving technical details of the actual constructions of biorthogonal spline-wavelets on periodic domains from [8] employed here, we collect only those properties which are relevant in the present context:

Locality (L): The functions $\psi_{\lambda}$ are local; the widths of their support decrease with growing discretization level $|\lambda|$,

$$
\operatorname{diam}\left(\operatorname{supp} \psi_{\lambda}\right) \sim 2^{-|\lambda|}
$$

Cancellation property (CanP): There exists an integer $\tilde{d}$ such that

$$
\left\langle v, \psi_{\lambda}\right\rangle \lesssim 2^{-|\lambda|(\tilde{d}+1)} \text { ess } \sup _{x \in \operatorname{supp} \psi_{\lambda}}\left|v^{(\tilde{d})}(x)\right|,
$$

where $v^{(\tilde{d})}$ denotes the weak $\tilde{d}$-th weak derivative of $v$. We say that $\Psi$ has $\tilde{d}$ vanishing moments: integration of $v$ against a wavelet has the effect of taking an $\tilde{d}$-th order difference, annihilating the smooth part of $v$. It is this property which entails quasi-sparse representations of a wide class of operators which is essential for a fast application of the operators $A$ and $R$ in Section 5.2, see [6,7].

To realize this property, one typically constructs $\Psi$ in such a way that it possesses a dual or biorthogonal basis $\tilde{\Psi} \subset \mathcal{H}^{\prime}$ such that the multiresolution spaces $\tilde{S}_{j}:=\operatorname{span}\left\{\tilde{\psi}_{\lambda}:|\lambda|<j\right\}$ contain all polynomials of order $\tilde{d}$. Here dual basis means that $\left\langle\psi_{\lambda}, \tilde{\psi}_{v}\right\rangle=\delta_{\lambda, v}, \lambda, v \in \mathbb{I}$.

Riesz basis property (R): Every $v \in \mathcal{H}$ has a unique expansion in terms of $\Psi$,

$$
v=\sum_{\lambda \in \mathbb{I}} v_{\lambda} \psi_{\lambda}=: \mathbf{v}^{T} \Psi, \quad \mathbf{v}:=\left(v_{\lambda}\right)_{\lambda \in \mathbb{I}},
$$

and its expansion coefficients satisfy the following norm equivalence: There exist finite positive constants $c_{\mathcal{H}}, C_{\mathcal{H}}$ such that

$$
\mathcal{C}_{\Psi}\|\mathbf{v}\|_{\ell_{2}(\mathbb{I})} \leq\left\|\mathbf{v}^{T} \Psi\right\|_{\mathcal{H}} \leq C_{\Psi}\|\mathbf{v}\|_{\ell_{2}(\mathbb{I})}, \quad \mathbf{v} \in \ell_{2}(\mathbb{I})
$$

where $\|\mathbf{v}\|_{\ell_{2}(\mathbb{I})}:=\left(\sum_{\lambda \in \ell_{2}(\mathbb{I})}\left|v_{\lambda}\right|^{2}\right)^{1 / 2}$ and $\ell_{2}(\mathbb{I})$ contains all infinite sequences of vectors for which $\|\mathbf{v}\|_{\ell_{2}(\mathbb{I})}<\infty$. In other words, die Riesz basis property means that wavelet expansions induce isomorphisms between certain function spaces and sequence spaces. This is a crucial requirement for mimicking problem formulations in infinite spaces as we intend to do to prepare for appropriate numerical schemes. Using duality arguments, one can show that (4.5) is equivalent to the existence of a biorthogonal collection of functions

$$
\tilde{\Psi}:=\left\{\tilde{\psi}_{\lambda}: \lambda \in \mathbb{I}\right\} \subset \mathcal{H}^{\prime} \quad \text { such that }\left\langle\psi_{\lambda}, \tilde{\psi}_{\mu}\right\rangle=\delta_{\lambda, \mu}, \lambda, \mu \in \mathbb{I},
$$

which is a Riesz basis in $\mathcal{H}^{\prime}$, see, e.g., [8].

We will systematically use the following shorthand notation. We will view $\Psi$ both as in (4.1) as a collection of functions as well as an infinite column vector containing all 
functions, always assembled in some fixed order. For a countable collection of functions $\Theta$ and some single function $\sigma$, the term $\langle\Theta, \sigma\rangle$ is to be understood as the column vector with entries $\langle\theta, \sigma\rangle$, and $\langle\sigma, \Theta\rangle$ as a row vector. For two collections $\Theta, \Sigma$, the quantity $\langle\Theta, \Sigma\rangle$ is then a possibly infinite matrix with entries $(\langle\theta, \sigma\rangle)_{\theta \in \Theta, \sigma \in \Sigma}$ for which $\langle\Theta, \Sigma\rangle=$ $\langle\Sigma, \Theta\rangle^{T}$. This implies for a possibly infinite matrix $\mathbf{C}$ that $\langle\mathbf{C} \Theta, \Sigma\rangle=\mathbf{C}\langle\Theta, \Sigma\rangle$ and $\langle\Theta, \mathbf{C} \Sigma\rangle=$ $\langle\Theta, \Sigma\rangle \mathbf{C}^{T}$. In this notation, the expansion coefficients in (4.4) can explicitly be expressed as $\mathbf{v}^{T}=\langle v, \tilde{\Psi}\rangle$. Moreover, the biorthogonality or duality conditions (4.6) can be reexpressed as $\langle\Psi, \tilde{\Psi}\rangle=\mathbf{I}$ with the infinite identity matrix.

For a biinfinite matrix $\mathrm{C}: \ell_{2}(\mathbb{I}) \rightarrow \ell_{2}(\mathbb{I})$, we denote its operator norm as

$$
\|\mathbf{C}\|:=\|\mathbf{C}\|_{\ell_{2}(\mathbb{I}) \rightarrow \ell_{2}(\mathbb{I})}:=\sup _{\|\mathbf{w}\|_{\ell_{2}(\mathbb{I})} \leq 1}\|\mathbf{C w}\|_{\ell_{2}(\mathbb{I})} .
$$

\subsection{Wavelet Representation of Operators}

Now we are ready to derive wavelet representations of operators in terms of the wavelet basis $\Psi$ with dual basis $\tilde{\Psi}$. Let $A: \mathcal{H} \rightarrow \mathcal{H}^{\prime}$ be the linear operator introduced in Corollary 3.4 and $R: L_{2}(\Omega) \rightarrow\left(L_{2}(\Omega)\right)^{\prime} \cong L_{2}(\Omega)$ the Riesz operator defined before (3.19). We now derive a wavelet representation for problem (3.19).

Any image $A w \in \mathcal{H}^{\prime}$ can naturally be expanded with respect to $\tilde{\Psi}$ as $A w=\langle A w, \Psi\rangle \tilde{\Psi}$. Representing $w \in \mathcal{H}$ in its expansion $w=\mathbf{w}^{T} \Psi$ with respect to $\Psi$ and inserting this in the previous expression yields

$$
A w=\mathbf{w}^{T}\langle A \Psi, \Psi\rangle \tilde{\Psi}=(\langle\Psi, A \Psi\rangle \mathbf{w})^{T} \tilde{\Psi} .
$$

Lemma 4.1. The wavelet representation of $A: \mathcal{H} \rightarrow \mathcal{H}^{\prime}$ with respect to the bases $\Psi, \tilde{\Psi}$ of $\mathcal{H}, \mathcal{H}^{\prime}$ is given by

$$
\mathbf{A}:=\langle\Psi, A \Psi\rangle, \quad A w=(\mathbf{A w})^{T} \tilde{\Psi},
$$

i.e., the expansion coefficients of Aw are obtained by applying the biinfinite matrix $\mathbf{A}=\langle\Psi, A \Psi\rangle$ to the coefficient vector $\mathbf{w}$ of $w$. Moreover, $\mathbf{A}: \ell_{2}(\mathrm{I}) \rightarrow \ell_{2}(\mathrm{I})$ is boundedly invertible, i.e.,

$$
c_{A} c_{\Psi}^{2}\|\mathbf{v}\|_{\ell_{2}(\mathbb{I I})} \leq\|\mathbf{A v}\|_{\ell_{2}(\mathbb{I})} \leq C_{A} C_{\Psi}^{2}\|\mathbf{v}\|_{\ell_{2}(\mathbb{I})} \text {. }
$$

Analogously, we obtain that $\mathbf{R}: \ell_{2}(\mathbb{I}) \rightarrow \ell_{2}(\mathbb{I})$ defined by

$$
\mathbf{R}=\langle\Psi, R \Psi\rangle
$$

is symmetric positive definite and bounded; the latter follows from the continuous embedding (3.16) by

$$
\begin{aligned}
(\mathbf{R v}, \mathbf{w})_{\ell_{2}(\mathbb{I})} & =(v, w)_{L_{2}(\Omega)} \leq\|v\|_{L_{2}(\Omega)}\|w\|_{L_{2}(\Omega)} \\
& \leq c_{e}^{2}\|v\|_{\mathcal{H}}\|w\|_{\mathcal{H}} \leq c_{e}^{2} C_{\Psi}^{2}\|\mathbf{v}\|_{\ell_{2}(\mathbb{I})}\|\mathbf{w}\|_{\ell_{2}(\mathbb{I})} .
\end{aligned}
$$

Since the inverse direction $\|v\|_{\mathcal{H}} \lesssim\|v\|_{L_{2}(\Omega)}$ in general does not hold, $\mathbf{R}$ is usually not coercive. However, we can establish coercivity on a set where the Rayleigh quotient is 
bounded. In fact, if we assume the existence of a constant $K \in \mathbb{R}$ such that the Rayleigh quotient satisfies

$$
\mu(\mathbf{v}):=\frac{(\mathbf{A v}, \mathbf{v})_{\ell_{2}(I)}}{(\mathbf{R} \mathbf{v}, \mathbf{v})_{\ell_{2}(I)}} \leq K,
$$

then it follows that

$$
(\mathbf{R v}, \mathbf{v})_{\ell_{2}(\mathbb{I})} \geq \frac{1}{K}(\mathbf{A v}, \mathbf{v})_{\ell_{2}(\mathbb{I})} \geq \frac{c_{A} c_{\Psi}^{2}}{K}\|\mathbf{v}\|_{\ell_{2}(I)}^{2} .
$$

Thus, we have established the following result.

Lemma 4.2. If there exists a constant $K \in \mathbb{R}$ such that (4.13) holds, the Riesz operator $\mathbf{R}$ in wavelet coordinates is boundedly invertible with bounds provided by (4.12) and (4.14).

\subsection{Compression of Operators in Wavelet Representation}

An essential ingredient of the solution algorithm for (3.19) will be that the operators A and $\mathbf{R}$ in terms of a wavelet basis derived from the bilinear form $a(\cdot, \cdot)$ and $(\cdot, \cdot)_{L_{2}(\Omega)}$, respectively, fulfil certain Lipschitz and compression properties, see [6] for a derivation of the essential notions and [26] for details in the present context. The Lipschitz properties for $\mathbf{A}, \mathbf{R}$ follow immediately from the boundedness (4.10) and (4.12).

It was shown in [26] that for the situation at hand, it suffices for our problem (3.19) to prove the following compression estimate for wavelets $\psi_{\lambda}, \lambda \in \mathbb{I}$ (except for the coarsest level consisting of generator functions indexed by $\mathbb{I}_{j_{0}}$ ).

Theorem 4.1. The entries of $\mathbf{A}, \mathbf{R}$ satisfy

$$
\left\langle A \psi_{v}, \psi_{\lambda}\right\rangle \lesssim 2^{-\sigma|| \lambda|-| v||}, \quad\left\langle R \psi_{v}, \psi_{\lambda}\right\rangle \lesssim 2^{-\sigma|| \lambda|-| v||}
$$

with respect to the wavelet basis $\Psi$ of $H_{\text {per }}^{1}(\Omega)$, for all $\lambda \in \mathbb{I} \backslash \mathbb{I}_{j_{0}}$ and $v \in \mathbb{I}$ with a parameter $\sigma>\gamma>1$ in order to obtain a suitable decay parameter $\gamma>1$ in the compression according to Assumption 2 in [7].

Proof. We briefly sketch the proof to bring out the main mechanisms. Firstly, $A, R$ are local operators meaning that $\langle A u, v\rangle=a(u, v)=0$ for all $u, v \in H_{p e r}^{1}(\Omega)$ as soon as the interiors of the supports of $u, v$ do not intersect, and correspondingly for $R$. To achieve (4.15), we estimate $A u$ in a possibly high Sobolev norm. For $u \in H_{p e r}^{t}(\Omega)$ and $t \geq 2$, one has $\int_{\Omega}\left(\frac{\partial}{\partial x_{e}} u\right)\left(\frac{\partial}{\partial x_{e}} v\right) d x=-\int_{\Omega}\left(\frac{\partial^{2}}{\partial x_{e}^{2}} u\right) v d x$. Thus, for $u \in H_{\text {per }}^{2}(\Omega)$, the operator $A$ coincides with the operator $H:=E_{g}+E_{s}+\hat{H}_{k i n}+\hat{H}_{a t t r}+\hat{H}_{\text {dis }}$ since $\langle A u, v\rangle=(H u, v)_{L_{2}(\Omega)}$ and $\langle A u, v\rangle=$ $(A u, v)_{L_{2}(\Omega)}$ for all $v \in L_{2}(\Omega)$ if $A u \in L_{2}(\Omega) \cong\left(L_{2}(\Omega)\right)^{\prime}$ by definition of the dual pairing in terms of a Gelfand triple (3.15). Similar to the proof of (3.9) in Theorem 3.2, we can now 
estimate

$$
\begin{aligned}
\|A u\|_{L_{2}(\Omega)} \leq & \max \left\{E_{g}+E_{S}, \frac{\hbar^{2}}{2 m_{e}^{*}}, \frac{\hbar^{2}}{2 m_{e}^{*}}, \max _{i=1, \ldots, M}\left\{\left|\operatorname{Ran}_{\text {dis,e }}(i)\right|,\left|\operatorname{Ran}_{d i s, h}(i)\right|\right\}, 1\right\} \\
& \times \int_{\Omega}\left(4 u+\frac{\partial^{2}}{\partial x_{e}^{2}} u+\frac{\partial^{2}}{\partial x_{h}^{2}} u\right)^{2} d x \\
\lesssim & \sum_{|\alpha| \leq 2}\left\|D^{\alpha} u\right\|_{L_{2}(\Omega)}^{2}=:\|u\|_{H^{2}(\Omega)}^{2} .
\end{aligned}
$$

That means, by Remark 7.2 in [6], the compression property (4.15) for A is fulfilled with $\sigma=2>1$ for a wavelet basis of $H_{p e r}^{1}(\Omega)$ of the above form.

Analogously, we have to estimate $R u$ in a possibly high Sobolev norm. By the definition of $R$, one has for $H_{\text {per }}^{s+1}(\Omega)$ that $\|R u\|_{H^{s-1}(\Omega)}=\|u\|_{H^{s-1}(\Omega)}$ implying $\|R u\|_{H^{s-1}(\Omega)} \lesssim$ $\|u\|_{H^{s+1}(\Omega)}$ for all $s \in \mathbb{N}_{0}$. That is, again by Remark 7.2 in [6] the compression property (4.15) with respect to $\mathbf{R}$ is fulfilled with $\sigma=2>1$ depending on the regularity of the wavelet basis of $H_{\text {per }}^{1}(\Omega)$. Finally, by Proposition 5.5.5 in [26], we obtain a suitable decay parameter $\gamma>1$.

\section{An Adaptive Wavelet Method for the Eigenvalue Problem}

\subsection{An Idealized Scheme in Infinite Wavelet Coordinates}

Next we state a basic iteration scheme which is yet in an idealized form since it is still in infinite wavelet coordinates. In view of the properties of $\mathbf{A}$ stated in Lemma 4.1, the operator

$$
\mathbf{P}:=\frac{c_{A} c_{\Psi}^{2}+C_{A} C_{\Psi}^{2}}{2} \mathbf{I}
$$

is a bijective and self-adjoint preconditioner for $\mathbf{A}$ such that

$$
\left\|\mathbf{I}-\mathbf{P}^{-1} \mathbf{A}\right\|_{\mathbf{A}} \leq \varrho<1
$$

where $\|\mathbf{v}\|_{\mathbf{A}}^{2}:=(\mathbf{A v}, \mathbf{v})_{\ell_{2}(I)}$ for $\mathbf{v} \in \ell_{2}(\mathbb{I})$ defines a norm on $\ell_{2}(\mathbb{I})$ and $\varrho:=\frac{C_{A} C_{\Psi}^{2}-c_{A} c_{\Psi}^{2}}{C_{A} C_{\Psi}^{2}+c_{A} c_{\Psi}^{2}}$. Now we can state an idealized iteration in terms of operators in a perturbed realizable form.

Definition 5.1. Given an initial guess $\mathbf{v}^{(0)} \in \ell_{2}(\mathbb{I})$ with $\mathbf{v}^{(0)} \neq 0$, the perturbed preconditioned inverse iteration (PPINVIT) generates a sequence of vectors $\left(\mathbf{v}^{(n)}\right)_{n \geq 0}$ and associated Rayleigh quotients $\left(\mu^{(n)}\right)_{n \geq 0}$ by

$$
\begin{aligned}
\mathbf{v}^{(n+1)} & =\mathbf{v}^{(n)}-\mathbf{P}^{-1}\left(\mathbf{A} \mathbf{v}^{(n)}-\mu\left(\mathbf{v}^{(n)}\right) \mathbf{R} \mathbf{v}^{(n)}\right)+\boldsymbol{\zeta}^{(n)} \\
\mu^{(n+1)} & =\mu\left(\mathbf{v}^{(n+1)}\right),
\end{aligned}
$$

where $\mu(\mathbf{v})=\frac{(\mathbf{A v}, \mathbf{v})_{\ell_{2}(I)}}{(\mathbf{R}, \mathbf{v})_{\ell_{2}(I)}}$ denotes the generalized Rayleigh quotient of $\mathbf{v}$ and $\left(\boldsymbol{\xi}^{(n)}\right)_{n \geq 0}$ are perturbations with $\xi^{(n)} \in \ell_{2}(\mathbb{I})$ for all $n \geq 0$. 
This scheme is the one from [20] generalized to the situation of infinite matrices. In principle, the convergence proof of PPINVIT can be found in [20] or [19] since it can be generalized to the infinite case, cf. [31]. Details are provided in [21,26].

\subsection{The Perturbed Realizable Scheme}

We are finally in the position to formulate our algorithm with approximations of $\mathbf{A}, \mathbf{R}$ defined as follows.

Definition 5.2. For $\mathbf{A v}$ and $\mathbf{R v}$ we define its approximations $\mathbf{A}_{\varepsilon}(\mathbf{v})$ and $\mathbf{R}_{\varepsilon}(\mathbf{v})$ for any $\mathbf{v} \in \ell_{2}(\mathbb{I})$ and $\varepsilon>0$ such that

$$
\left\|\mathbf{A}_{\varepsilon}(\mathbf{v})-\mathbf{A v}\right\|_{\ell_{2}(\mathbb{I})} \leq \varepsilon\|\mathbf{v}\|_{\ell_{2}(\mathbb{I})}, \quad\left\|\mathbf{R}_{\varepsilon}(\mathbf{v})-\mathbf{R} \mathbf{v}\right\|_{\ell_{2}(\mathbb{I})} \leq \varepsilon\|\mathbf{v}\|_{\ell_{2}(\mathbb{I})} .
$$

Moreover, we define the resulting perturbed Rayleigh quotient as

$$
\mu_{\varepsilon}(\mathbf{v}):=\frac{\left(\mathbf{A}_{\varepsilon}(\mathbf{v}), \mathbf{v}\right)_{\ell_{2}(\mathrm{II})}}{\left(\mathbf{R}_{\varepsilon}(\mathbf{v}), \mathbf{v}\right)_{\ell_{2}(\mathrm{I})}} .
$$

Note that this definition differs from the corresponding definition of the approximations in [31] in the way that we have the same norms on the right-hand side of the estimates above here. Obviously, this will result in slightly modified proofs of the corresponding statements in [31].

The adaptive operator applications needed here are performed in the context of a scheme APPLY on tree-structured index sets, see [6] for the theoretical development involving nonlinear local operators. This has been implemented based on piecewise polynomials in [33] for two-dimensional problems and in [28] for wavelets of tensor product structure in arbitrary dimensions.

Our final realizable version of PPINVIT based on adaptive approximate operator applications in wavelet discretization is now as follows, see $[21,26]$ for the problemdependent specifications of the constants $c_{0}, c_{2}, c_{3}$. For a given user-specified tolerance $\tau$ and input vector $\mathbf{v}$, it outputs the eigenstate $\mathbf{u}$.

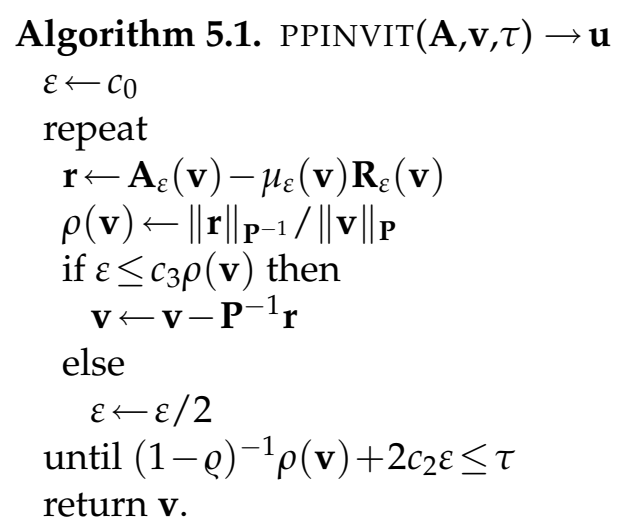


Concerning the convergence of Algorithm 5.1, our main result similar to the one in [31] is the following [21,26].

Theorem 5.2. For an arbitrary initial guess $\mathbf{v}^{(0)} \neq \mathbf{0}$ with corresponding Rayleigh quotient $\mu(\mathbf{v})<\mu_{2}$, Algorithm 5.1 has the following properties:

1. In each iteration step, the Rayleigh quotient decays like

$$
\frac{\mu^{\prime}-\mu_{1}}{\mu_{2}-\mu^{\prime}} \leq q^{2} \frac{\mu-\mu_{1}}{\mu_{2}-\mu^{\prime}}, \quad q:=1-(1-\tilde{\gamma}) \frac{\mu_{2}-\mu_{1}}{\mu_{2}}<1
$$

where $\mu, \mu^{\prime}$ are the Rayleigh quotients of the vectors one iteration step earlier and later, respectively, $\mu_{1}, \mu_{2}$ are the smallest and second smallest eigenvalues of $\mathbf{A}$, and $\tilde{\gamma}:=\varrho+\gamma_{\xi}<1$ with $\gamma_{\xi}$ a constant bounding the perturbations $\xi$;

2. the residual $\rho(\mathbf{v})$ decreases in each iteration step;

3. the accuracy $\varepsilon$ is proportional to the residual $\rho(\mathbf{v})$ in each step if $\varepsilon$ is small enough;

4. the maximal accuracy \& needed for the approximate application of $\mathbf{A}$ and $\mathbf{R}$ remains bounded by

$$
\varepsilon \gtrsim \max \{\tau, \rho(\mathbf{v})\} ;
$$

5. the final output vector $\mathbf{u}$ fulfills $\rho(\mathbf{u}) \leq \tau$.

It is very likely that Algorithm 5.1 is asymptotically optimal according to this notion known in the wavelet context from, e.g., [5], if one uses the optimal application scheme derived in $[21,26]$. The similarity of the PPINVIT scheme above with the GROW algorithm from [13] in the context of partial differential equations and the numerical results in Section 6 corroborate this assertion; in particular, it seems that we do not need an additional coarsening of the iterands.

\section{Numerical Results}

The numerical results have been developed in [26] based on the AWM toolbox developed in [33] for the adaptive wavelet part combined with the programs from [31] and the matrix eigenvalue solver BLOPEX from [18].

Our setup is the following. We will employ periodized and isotropic tensorized scaled B-spline wavelets of order $d=2$, i.e., consisting of continuous piecewise linear polynomials, with vanishing moments $\tilde{d}=2$ as the underlying wavelet basis of $H_{\text {per }}^{1}\left((0, L)^{2}\right)$. We fix in each realization the constants

$$
\frac{m_{e} *}{m_{e}}:=0.067, \quad \frac{m_{h} *}{m_{e}}:=0.8, \quad \hat{\gamma} \hat{R}:=80 a_{0}, \quad E_{g}:=0,
$$




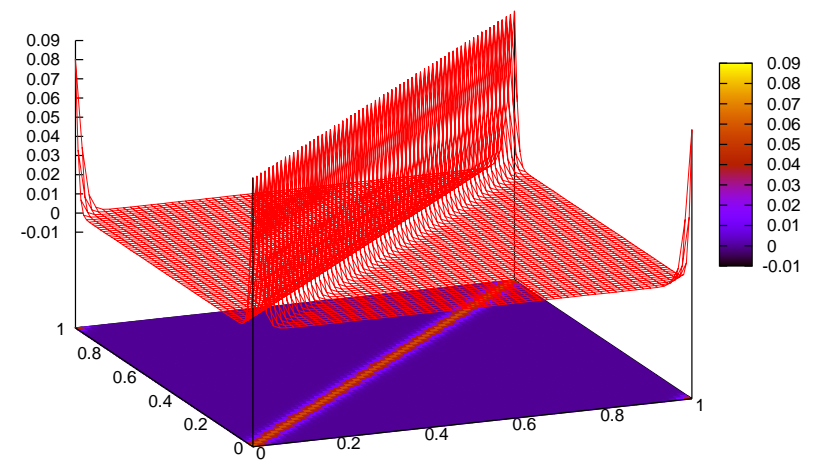

(a) : Eigenfunction

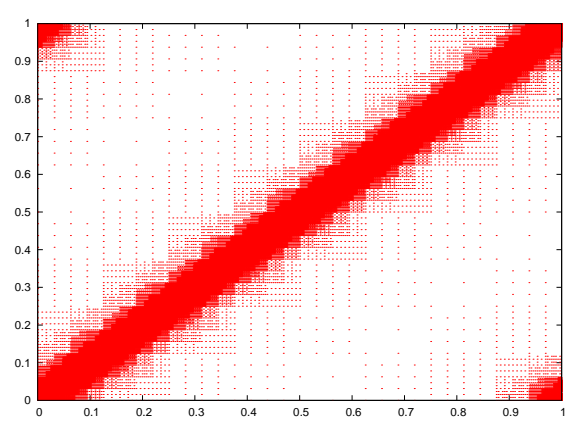

(b) : Active Wavelets

Figure 2: Eigenfunction without disorder with respect to wire length $L=15120 a_{0}$ (left) and used active wavelets after some PPINVIT iteration steps (right). Each dot corresponds to the center of the support of a used wavelet. That is, more dots in one area correspond to more used degrees of freedom in this area.

with $a_{0}$ the Bohr radius. For the computations and the subsequent plots, we always transform the problem onto the unit cube $[0,1]^{2}$.

We set the shift in (3.8) to $E_{s}=0.001$ with respect to the calculations without disorder and possibly choose a larger shift when it comes to calculations with the disorder term, depending on the disorder potential itself. Moreover, we scale the eigenvalue problem with a scaling factor $10^{5}$ in order to scale the eigenvalues. That is, we consider the eigenvalue problem of finding the smallest eigenvalue $\tilde{E}$ and corresponding eigenfunction $u \in H_{\text {per }}^{1}\left((0, L)^{2}\right)$ such that $\tilde{a}(u, v)=\tilde{E}(u, v)_{L_{2}\left((0, L)^{2}\right)}$ for all $v \in H_{\text {per }}^{1}\left((0, L)^{2}\right)$ with $\tilde{a}(u, v):=10^{5}\left[\hat{a}(u, v)+0.001(u, v)_{L_{2}\left((0, L)^{2}\right)}\right]$ and $\tilde{E}=10^{5}(\hat{E}+0.001)$ from (3.6). Obviously, the original eigenvalue can now be computed setting $\hat{E}=10^{-5} \tilde{E}-0.001$.

Recall from Theorem 4.1 that the decay parameter $\gamma$ for $\mathbf{A}, \mathbf{R}$ which enters into the APPLY scheme has been proven to staisfy $\gamma<2$. However, this choice is much too pessimistic, as observed already in [33], so that we have also chosen $\gamma=4$ for the application of $\mathbf{A}$ and $\mathbf{R}$ in the following.

The initial vector needs to be small enough in the sense that its Rayleigh quotient needs to be smaller than the second smallest eigenvalue, i.e., $\mu\left(v^{(0)}\right)<\mu_{2}$. To achieve this, we generate an initial vector by solving a comparatively small eigenvalue problem on a fixed index set with the iterative eigenvalue solver BLOPEX developed in [18]. 


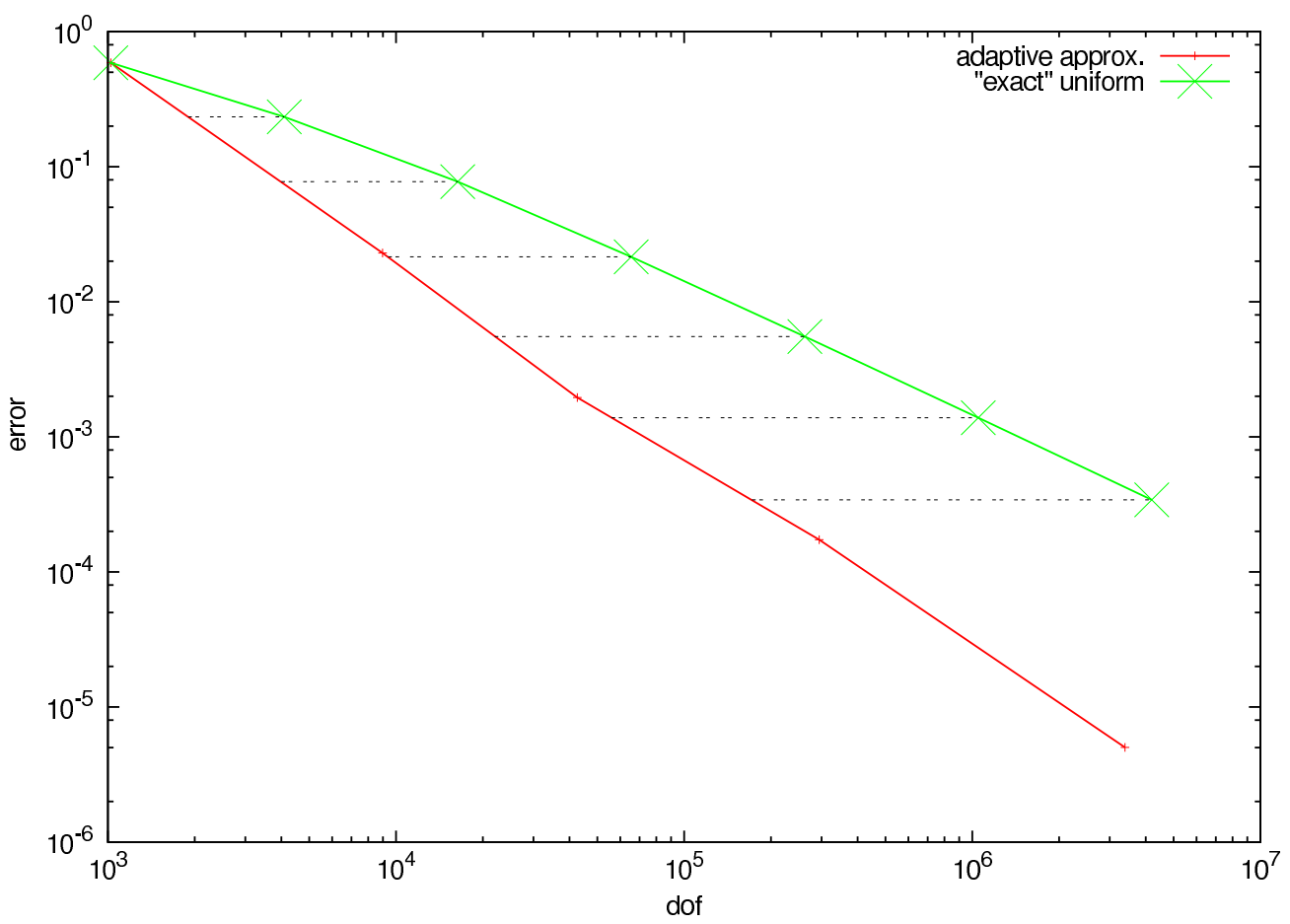

Figure 3: 'Exact' eigenvalues with respect to uniform grids in comparison to approximations by the adaptive PPINVIT scheme with $L=15120 a_{0}$.

\section{Adaptivity}

First we want to investigate the adaptivity of our scheme. Figure 2 shows the approximation of an eigenfunction with respect to the smallest eigenvalue without disorder and the corresponding distribution of the degrees of freedom which are used in the calculation. Here, we plot the active wavelets with respect to an intermediate iterand after some PPINVIT steps. Each dot corresponds to the center of the support of a used wavelet respectively scaling function. If one takes a look at the eigenfunction, one can easily see that one would likes to have the highest resolution along the diagonal and at the corners, and a low resolution elsewhere. Due to the translational invariance of our system, the solution does only depend on the difference between the electron and hole coordinate (evaluated taking into account the periodic boundary conditions) and has the expected exponentially decaying form of a 1s function. One can see that exactly such a desired distribution of degrees of freedom is generated by the adaptive wavelet scheme, see Figure 2 . 


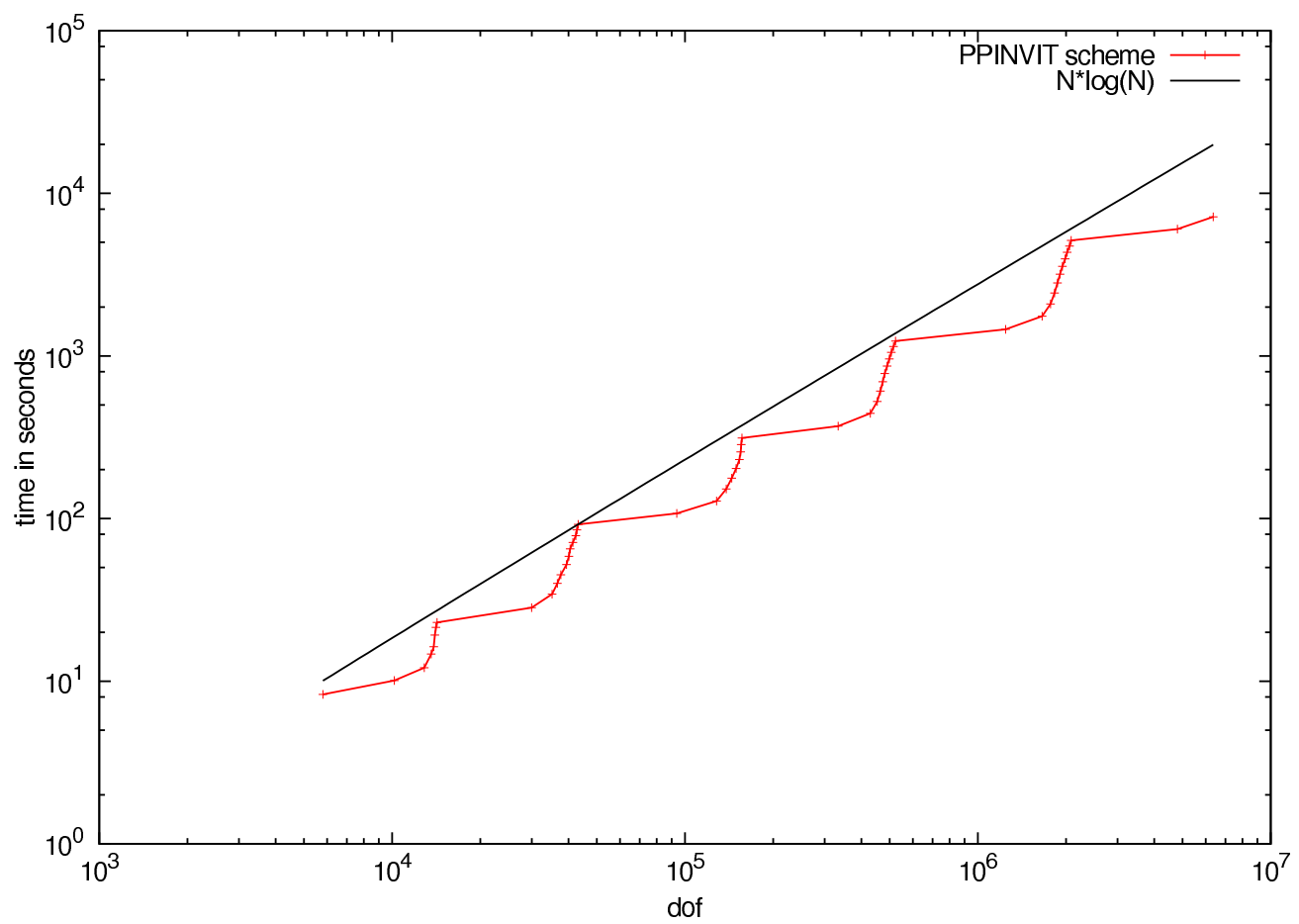

Figure 4: Computational complexity of the PPINVIT scheme.

\section{Performance of the PPINVIT Scheme}

In order to compare the approximation error with respect to uniform grids and adaptive grids, we set the solution on a uniform grid in relation with an approximation on an adaptive grid with comparably size, as it is done in Figure 3. Each big cross corresponds to the approximation error of the smallest eigenvalue with respect to a fixed uniform grid. One can see that the approximation on an adaptively generated grid in connection with the PPINVIT scheme yields much more accurate approximations then the best possible uniform approximation with the same number of degrees of freedom. Consequently, one needs much less degrees of freedom in order to achieve the same accuracy.

Another important aspect for the efficiency of adaptive solution methods is the computational effort of the scheme. Figure 4 shows the computational complexity of the adaptive scheme by setting the used number of degrees of freedom in relation to time. Since the memory access of the elements in each involved vector can be performed in constant time, see [33], the time can be chosen as a measure for the number of arithmetic and sorting operations. One can see that the time needed to obtain an approximation of the eigenvalue with $N$ degrees of freedom increases like $N \log (N)$. That is, the adaptive scheme has an asymptotically near optimal computational complexity. It is important to note that one can get rid of the additional $\log$-factor by using quasi sorting based on 
binary binning instead of exact sorting in the involved vector approximation scheme.

\section{Results with Disorder}

Finally, we will briefly present some results with disorder of different strengths. Recalling the definition of the disorder potential (2.8) the strength of disorder is controlled by the variances of the random numbers. Since the explicit value of an eigenvalue of one single realization is not meaningful and differs for each disorder potential even if the variance $\sigma^{2}$ and number of steps $M$ in the potential are the same, we will only show the qualitative behavior of the eigenfunctions under the influence of a fixed disorder potential.

Some eigenfunctions for different strengths of disorder are shown in Figure 5. Here we have used the same set of random numbers and varied the scalings according to the variance $\sigma^{2}$. This choice has the advantage that one can more clearly observe the influence of the magnitude of the variance in some sense, since the potential, in principle, up to a scaling stays the same. We see that a weak disorder yields only a small change of the eigenfunction in comparison to the eigenfunction without disorder, and that a stronger disorder results in a significant modification of the eigenfunction, see, e.g., [24]. It can be observed that for stronger disorder the peak on the diagonal becomes more and more localized on a smaller spatial region. Further calculations have shown that, in principle, all realizations have a similar behavior, such that the eigenfunctions in Figure 5 illustrate the general effects qualitatively quite well.

In order to investigate on which area the peak becomes localized, one needs to take a look at the explicit realization of the disorder potential in more detail. According to Figure 6 it can be oberved that the peak is concentrated at the area which corresponds to the smallest value in the disorder potential. That is, the area corresponding to the smallest random number. From a physical point of view, this effect can be explained by the fact that the ground state describes the state with the smallest possible energy of the exciton. Obviously, a smaller potential results in a smaller energy of the exciton so that the probability to find an exciton in an area with small disorder potential is higher than elsewhere in the wire.

If we choose a larger number of steps $M$ in the disorder potential, i.e., a smaller length of each step, the effect itself stays the same. The difference is that using more steps $M$, i.e., decreasing the step length, reduces the localization effect so that one needs a stronger disorder in order to obtain a comparable localization.

Finally, in Figure 8 we have plotted the distribution of degrees of freedom for the different disorder potentials from Figure 5. Just as in the ideal case, cf. Figure 2, the resolution is much higher in the region of the peak than elsewhere so that the adaptive scheme resolves the peak appropriately. 


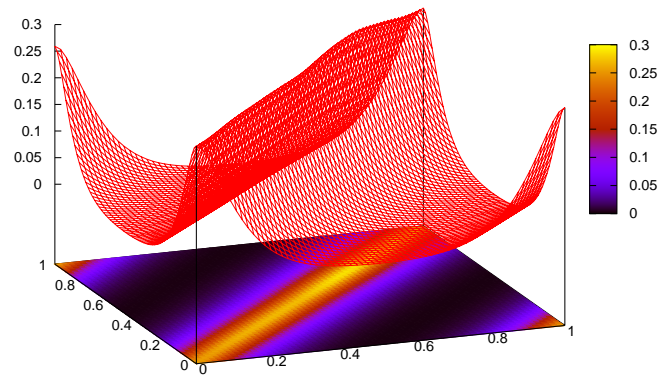

(a) $: \sigma=10^{-7} E_{h}$

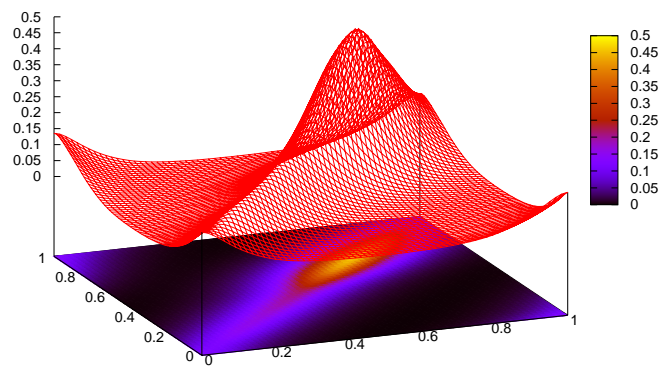

(c) : $\sigma=10^{-6} E_{h}$

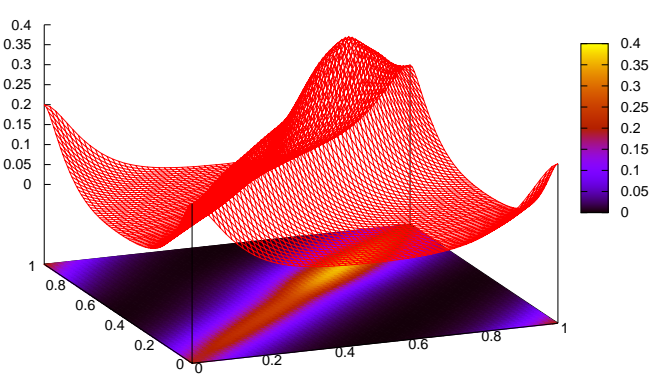

(b) : $\sigma=5 \times 10^{-7} E_{h}$

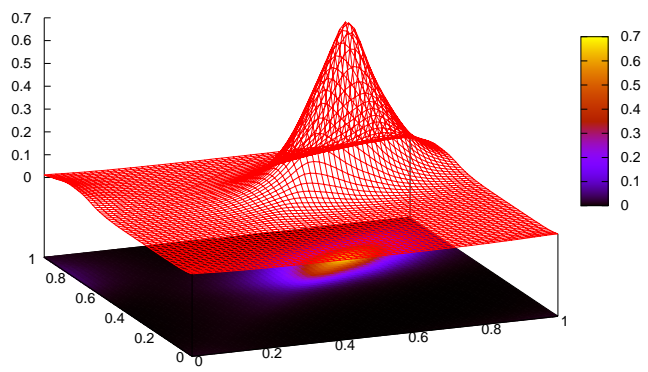

(d) $: \sigma=5 \times 10^{-6} E_{h}$

Figure 5: Eigenfunctions with respect to different potentials for $L=1890 a_{0}$ and $M=16$ steps in the disorder potential leading to a step length of $L / M \approx 118 a_{0}$. We have used the same set of random numbers but with different scalings according to the standard deviation $\sigma$. 


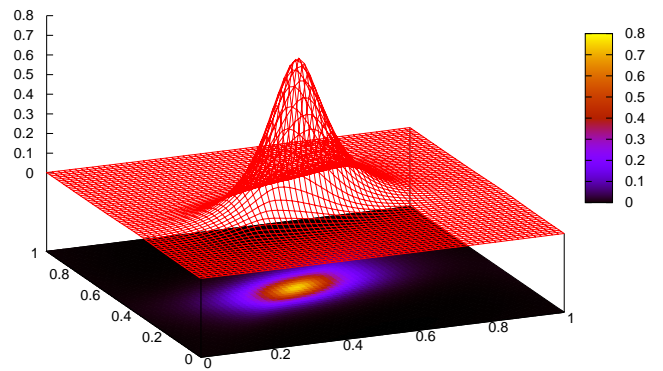

(a) : smallest value in the disorder potential in the area $x_{e}, x_{h} \in[0.4375,0.5)$

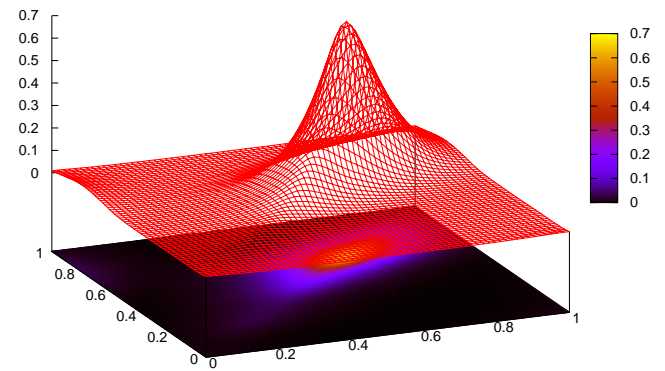

(b) : smallest value in the disorder potential in the area $x_{e}, x_{h} \in[0.625,0.6875)$

Figure 6: Eigenfunctions with respect to different realizations of disorder potentials for $L=1890 a_{0}$ and $M=16$ steps in the disorder potential, i.e., a step length of $L / M \approx 118 a_{0}$, with standard deviation $\sigma=5 \times 10^{-6} E_{h}$. The area corresponding to the smallest disorder potential is stated under the figures $a$ ) and $b$ ).

\section{Summary and Outlook}

We have presented a method for the adaptive numerical computation of eigenenergies and corresponding eigenstates with respect to the Schrödinger equation induced by a semiconductor quantum wire with and without disorder of the interface. It has been shown that the induced weak eigenvalue problem, respectively, its representation in terms of a suitable wavelet basis, can be modified in such a way that it fulfills all necessary conditions needed to apply an adaptive eigenvalue solver based on wavelets. Finally, in section 6 we have presented some numerical results and, in particular, focused on the adaptivity and the influence of the disorder. The results clearly demonstrate that one obtains much better results by using the introduced adaptive wavelet method instead of any method acting on uniform grids, cf. Figure 2 and 3.

In this paper, we have considered the eigenvalue problem determined by a quantum wire, that is, a quasi one-dimensional semiconductor nanostructure. A next step could be to consider higher dimensional structures which would entail a higher dimensional Schrödinger equation. This in turn means that one would need a higher dimensional wavelet basis. Since the used AWM-Toolbox from [33] is developed for the twodimensional setting, one cannot use this directly but would have to modify the data structures substantially. Instead, we can employ the new software package by Roland Pabel using tensorized wavelet bases in arbitrary dimensions [28,29].

Another modification considers the handling of the stochastic influence. In this paper, we have considered explicit realizations of the disorder potential and analyzed the 


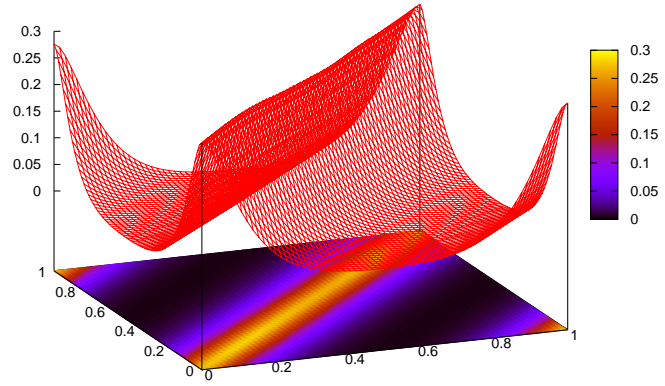

(a) : standard deviation $\sigma=10^{-6} E_{h}$

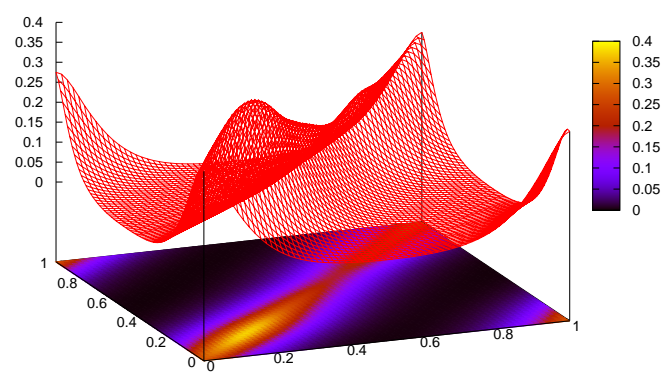

(c) : standard deviation $\sigma=10^{-5} E_{h}$

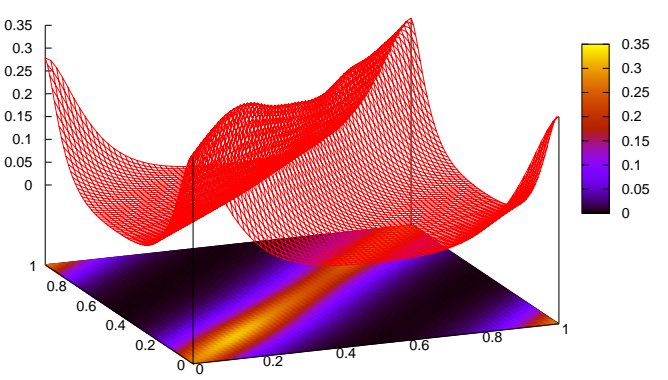

(b) : standard deviation $\sigma=5 \times 10^{-6} E_{h}$

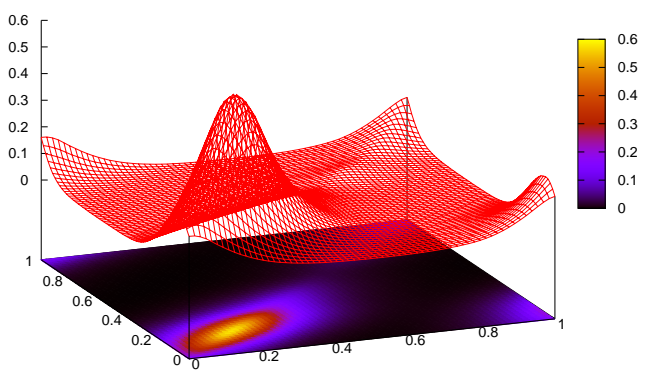

(d) : standard deviation $\sigma=5 \times 10^{-5} E_{h}$

Figure 7: Eigenfunctions with respect to different disorder potentials for $L=1890 a_{0}$ and $M=4096$ steps in the disorder potential, i.e., a step length of $L / M \approx 0.5 a_{0}$. We have used the same set of random numbers but with different scalings according to $\sigma$. 


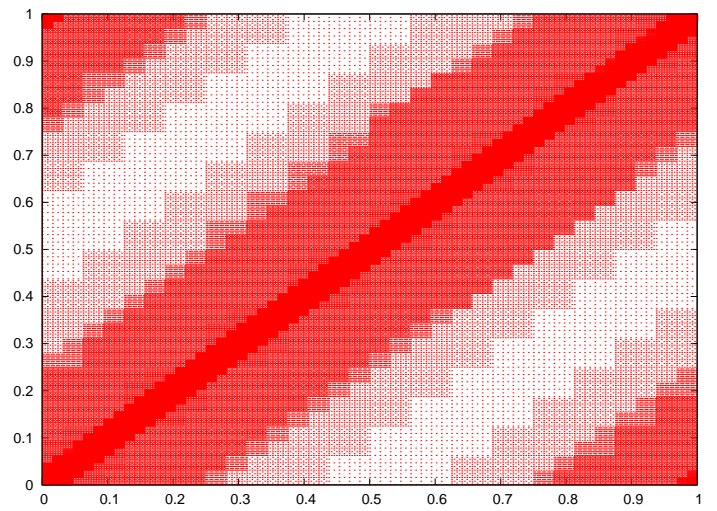

(a) : standard deviation $\sigma=10^{-7} E_{h}$

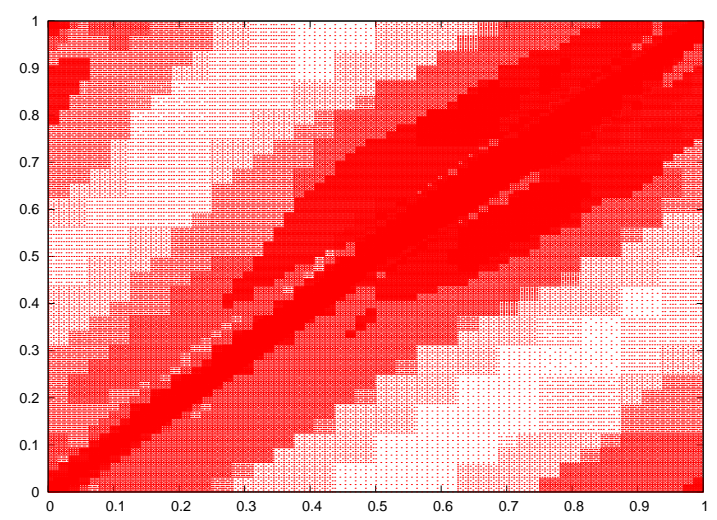

(c) : standard deviation $\sigma=10^{-6} E_{h}$

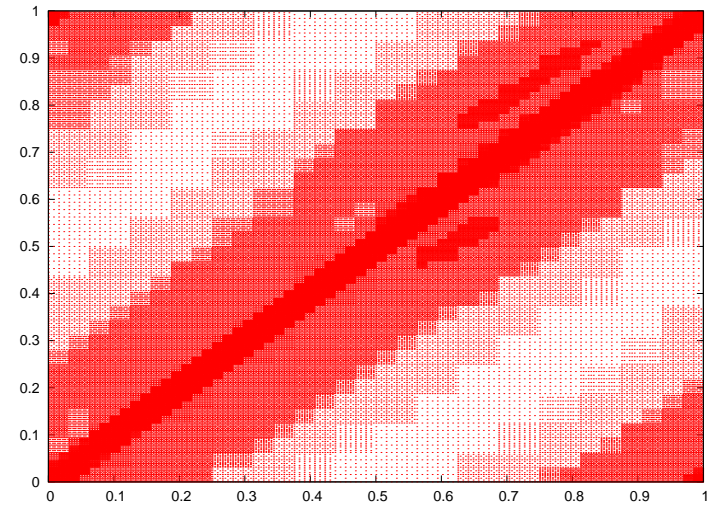

(b) : standard deviation $\sigma=5 \times 10^{-7} E_{h}$

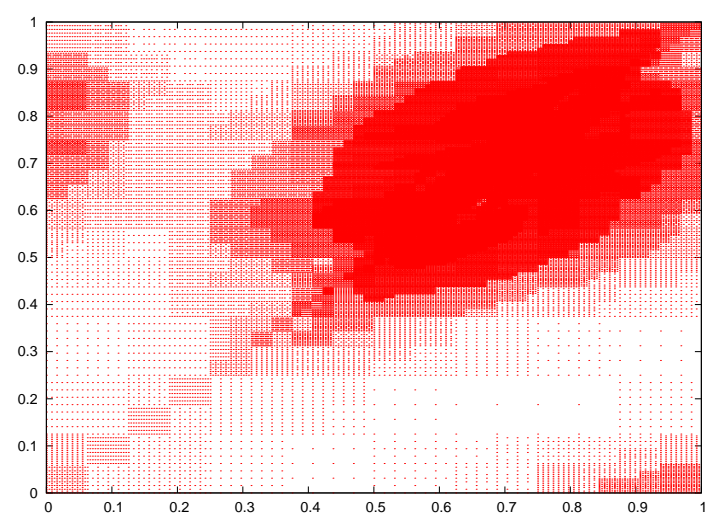

(d) : standard deviation $\sigma=5 \times 10^{-6} E_{h}$

Figure 8: Active wavelets for different disorder potentials for $L=1890 a_{0}$ and $M=16$ steps in the disorder potential, i.e., a step length of $L / M \approx 118 a_{0}$, after some PPINVIT iteration steps. Each dot corresponds to the center of the support of a used wavelet. That is, more dots in one area correspond to more used degrees of freedom in this area. 


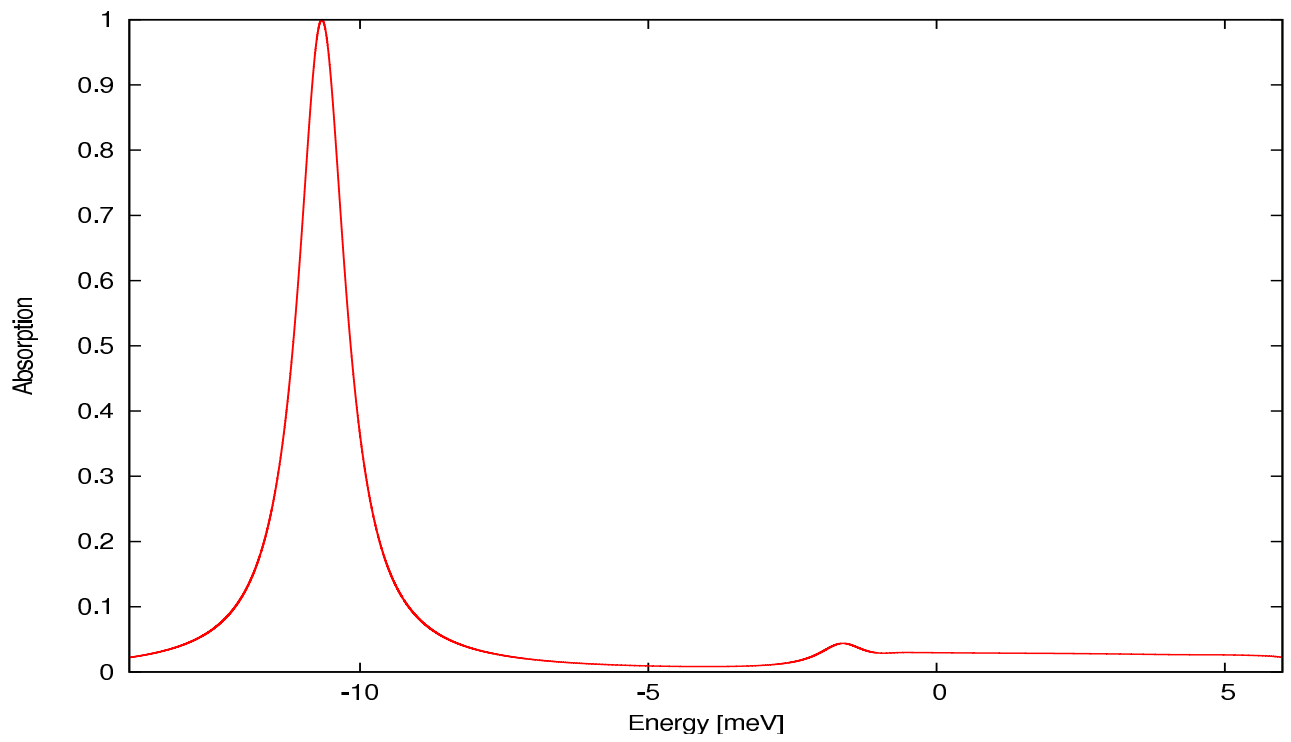

Figure 9: Example of an absorption spectra with respect to a quantum wire with length $L=40000 a_{0} \approx 2,116 \mu m$.

influence of the stochastic perturbation with respect to the eigenfunctions. In order to obtain quantitative results for the stochastic part, one would have to calculate several realizations and average characteristics. For handling such kind of problems, we could employ the method recently introduced in [16]. Here the original stochastic problem is transformed into a parametric deterministic one followed by low-rank approximations based on the pivoted Cholesky decomposition.

From a physical point of view, one is typically interested in, e.g., absorption spectra of certain nanostructures as shown in Figure 9. Such an absorption spectrum can be calculated with the aid of several eigenvalues or by solving the complete time-dependent equation for $p(x, t)$ and performing a Fourier transformation $[17,24]$. The PPINVIT scheme provides an approximation of the smallest eigenvalue and not of larger eigenvalues. It is possible to generalize the PPINVIT scheme to the case where the $m$ smallest eigenvalues are computed simultaneously by a subspace or block iteration method. Such a block version was already introduced in [20] for the case of finite matrices. Therefore, one could mimic this block version and consider a perturbed version similar as it is done in [31] for the present version of the PPINVIT scheme.

Another possibility to obtain the absorption spectra is via the polarization which in turn is given by the solution of the time dependent Schrödinger equation, see [24]. To this end, one could numerically solve the time dependent Schrödinger equation instead of the derived eigenvalue problem. This could be done by conventional time stepping schemes or, much more sophisticated, using the novel wavelet approach from [32] based on a weak space-time form of the parabolic equation. First steps in this direction have 
been made for the time dependent Schrödinger equation in preparation of [25].

\section{Acknowledgments}

We wish to thank J. Vorloeper and A. Zeiser for making their $\mathrm{C}^{++}$codes available to us, and for dicussions concerning the implementations. Angela Kunoth's research was supported in part by the Institute for Mathematics and its Applications (IMA) at the University of Minnesota with funds provided by the National Science Foundation (NSF). Torsten Meier's work is supported by the Deutsche Forschungsgemeinschaft (DFG).

\section{References}

[1] R.A. Adams and J.J.F. Fournier. Sobolev spaces. Academic press, second edition, 2003.

[2] S.D. Baranovskii and A.L. Efros. Sov. Phys. Semicond., 12:1328, 1978.

[3] S.L. Chuang. Physics of Optoelectronic Devices. John Wiley \& Sons, Inc., New York, 1995.

[4] A. Cohen. Numerical Analysis of Wavelet Methods. Studies in Mathematics and its Applications 32, Elsevier, 2003.

[5] A. Cohen, W. Dahmen, and R. DeVore. Adaptive wavelet methods for elliptic operator equations: Convergence rates. Math. Comp., 70(233):27-75, 2001.

[6] A. Cohen, W. Dahmen, and R. DeVore. Adaptive wavelet schemes for nonlinear variational problems. SIAM J. Numer. Anal., 41(5):1785-1823, 2003.

[7] A. Cohen, W. Dahmen, and R. DeVore. Sparse evaluation of compositions of functions using multiscale expansions. SIAM J. Math. Anal., 35(2):279-303, 2003.

[8] W. Dahmen. Wavelet and multiscale methods for operator equations. Acta Numerica, 6:55228, 1997.

[9] W. Dahmen, T. Rohwedder, R. Schneider, and A. Zeiser. Adaptive eigenvalue computation: Complexity estimates. Numer. Math., 110(3):277-312, 2008.

[10] R. Dautray and J.-L. Lions. Mathematical Analysis and Numerical Methods for Science and Technology, volume 2: Functional and Variational Methods. Springer, second edition, 1988.

[11] R. DeVore and A. Kunoth. Multiscale, Nonlinear and Adaptive Approximation. Springer Berlin Heidelberg, 2009.

[12] M. Fox. Quantum Optics: An Introduction. Oxford University Press, 2006.

[13] T. Gantumur, H. Harbrecht, and R. Stevenson. An optimal adaptive wavelet method without coarsening of the iterands. Math. Comp., 76(258):615-629, 2006.

[14] N. Gögh, P. Thomas, I. Kuznetsova, T. Meier, and I. Varga. Annalen der Physik, 18:905, 2009.

[15] W. Hackbusch. Elliptic Differential Equations: Theory and Numerical Treatment. Springer, 2003.

[16] H. Harbrecht and J. Li. A fast deterministic method for stochastic elliptic interface problems based on low-rank approximation. Research Report No. 2011-24, Seminar für Angewandte Mathematik, ETH Zürich, Switzerland, 2011.

[17] H. Haug and S.W. Koch. Quantum Theory of the Optical and Electronic Poperties of Semiconductors. World Scientific Publishing, Singapore, fourth edition, 2004.

[18] A.V. Knyazev, M.E. Argentati, I. Lashuk, and E.E. Ovtchinnikov. Block Locally Optimal Preconditioned Eigenvalue Xolvers (BLOPEX) in Hypre and PETSc. SIAM J. Sci. Comput., 29(5):2224-2239, 2007. 
[19] A.V. Knyazev and K. Neymeyr. Gradient flow approach to geometric convergence analysis of preconditioned eigensolvers. SIAM J. Matrix Anal. Appl., 31(2):621-628, 2009.

[20] V.A. Knyazev and K. Neymeyr. A geometric theory for preconditioned inverse iteration III: A short and sharp convergence estimate for generalized eigenvalue problems. Linear Algebra Appl., 358(1-3):95-114, 2003.

[21] A. Kunoth and Chr. Mollet. Adaptive computation of eigenvalues for the electronic Schrödinger equation based on wavelets. Manuscript in preparation, 2011.

[22] I. Kuznetsova, N. Gögh, J. Förstner, T. Meier, S.T. Cundiff, I. Varga, and P. Thomas. Phys. Rev. B, 81:075307, 2010.

[23] V. Mehrmann and A. Miedlar. Adaptive computation of smallest eigenvalues of self-adjoint elliptic partial differential equations. Numerical Linear Algebra, 18(3):387-409, 2011.

[24] T. Meier, P. Thomas, and S.W. Koch. Coherent Semiconductor Optics. Springer, 2007.

[25] C. Mollet. A Space-Time Adaptive Wavelet Method for the Electron-Hole Schrödinger Equation with Random Disorder. PhD thesis, Institut für Mathematik, Universität Paderborn, Germany, In preparation.

[26] Chr. Mollet. Excitonic Eigenstates in Disordered Semiconductor Quantum Wires: Adaptive Computation of Eigenvalues for the Electronic Schrödinger Equation Based on Wavelets. Shaker-Verlag, DOI: 10.2370/OND000000000098, 2011.

[27] Chr. Mollet, T. Meier, and A. Kunoth. Wavelet-based adaptive computations of the excitonic eigenstates of disordered semiconductor quantum wires. Accepted as poster presentation for 2nd International Workshop TaCoNa-Photonics, 2011.

[28] Chr. Mollet and R. Pabel. Efficient application of nonlinear stationary operators in adaptive wavelet methods. Manuscript, 2011.

[29] R. Pabel. Adaptive Wavelet Methods for PDE Constrained Nonlinear Elliptic Control Problems (working title). PhD thesis, Institut für Mathematik, Universität Paderborn, Germany, In preparation.

[30] W.H. Press, S.A. Teukolsky, W.T. Vetterling, and B.P. Flannery. Numerical Recipes: The Art of Scientific Computing. Cambridge University Press, third edition, 2007.

[31] T. Rohwedder, R. Schneider, and A. Zeiser. Perturbed preconditioned inverse iteration for operator eigenvalue problems with application to adaptive wavelet discretization. Adv. Comput. Math., 34(1):43-66, 2011.

[32] C. Schwab and R. Stevenson. Space-time adaptive wavelet methods for parabolic evolution problems. Math. Comp., 78(267):1293-1318, 2009.

[33] J. Vorloeper. Adaptive Wavelet Methoden für Operator Gleichungen - Quantitative Analyse und Softwarekonzepte (in German). VDI-Verlag, 2010.

[34] H. Yserentant. On the electronic Schrödinger equation. Lecture notes, Universität Tübingen, Germany, 2003.

[35] H. Zhu, Chen. Y., Song. S., and H. Hu. Erratum to: Symplectic and multi-symplectic wavelet collocation methods for two-dimensional Schrödinger equations. Applied Numerical Mathematics, 61:974-976, 2011.

[36] R. Zimmermann, F. Große, and E. Runge. Pure E Appl. Chem., 69:1179, 1997. 